\title{
High-Reliability and Low-Complexity Resource Allocation for Future Multicarrier Spread Spectrum Systems
}

\author{
Jia Shi ${ }^{1}$ \\ Zan Li ${ }^{1}$ \\ ${ }^{1}$ State Key Laboratory of Integrated \\ Services Networks, Xidian University, Xi'an, \\ Shaanxi, 710071, China. Email: \\ jiashi@xidian.edu.cn, zanli@xidian.edu.cn \\ $25 \mathrm{G}$ Innovation Center, University of Surrey, \\ Guildford, Surrey, GU1 2XH, UK.Email: \\ j.shi@surrey.ac.uk, p.xiao@surrey.ac.uk \\ ${ }^{3}$ Department of Communications \\ Engineering, Northwestern Polytechnical \\ University, Xi'an, China. Email: \\ liangwei@nwpu.edu.cn \\ ${ }^{4}$ Southwest University, Chong Qing, \\ 400715, China. Email: \\ penngaofeng@qq.com

\section{Correspondence} \\ Pei Xiao, 5G Innovation Center, University \\ of Surrey, Guildford, GU1 2XH, UK. \\ Email: p.xiao@surrey.ac.uk \\ Funding information \\ This work was sponsored by the UK \\ Engineering and Physical Sciences Research \\ Council (EPSRC) under grant number \\ EP/P008402/1. We would like to \\ acknowledge the support of the University \\ of Surrey $5 \mathrm{GIC}$ members for this work. This \\ work was also supported by the National \\ Natural Science Foundation of China under \\ Grant 61501356, Grant 61501354, Grant \\ 61631015.
}

In this paper, we investigate resource allocation in the multicarrier spread spectrum systems, especially in the multicell downlink multicarrier direct-sequence code division multiple-access (MC DS-CDMA) systems. The allocation of resources including subcarriers and spreading codes aims to maximize the system reliability, thereby resulting in the high-reliability mutlicarrier systems. For the sake of achieving low-complexity, we develop the novel resource allocation framework. We propose two resource allocation algorithms, which are the simplified heuristic subcarrier- and code-allocation (SHSC) algorithm and the enhanced heuristic subcarrier- and code-allocation (EHSC) algorithm. The two proposed algorithms can find the promising sub-optimum solutions to the mixed integer nonconvex resource allocation problem. The SHSC algorithm has lower complexity and demands less backhaul resources than the EHSC algorithm. In return, the EHSC algorithm performs better than the SHSC algorithm. Nevertheless, we show that both algorithms significantly outperform the existing algorithms, while approaching the optimal algorithm of high complexity. 


\section{KEYWORDS}

Multicarrier Spread Spectrum, MC DS-CDMA, multicell, resource allocation, high-reliability, low-complexity, bit error rate

\section{1 | INTRODUCTION}

Future wireless communication will demand higher and higher capacity, spectral efficiency and data rate to support high quality and diverse services. Whereas, massive growth of data service and mobile terminals will pose a huge challenge for future wireless systems with limited radio spectrum available. The use of direct-sequence spread spectrum aided multicarrier schemes can overcome the challenge for future wireless networks, and the development of robust transceivers that are able to transmit at high data rates with high bandwidth efficiency.

\section{1 | Related Works}

So far, code division multiple access (CDMA) technique has been adopted in 2G IS-95 and 3G WCDMA, and cdma2000, as well as IEEE $802.11 \mathrm{~b}$ standards, since it exploits the advantages of configuration flexibility, high capacity, and robustness to multipath effects [1,2]. By contrast, orthogonal frequency multiple access (OFDM) is a famous mutlicarrier modulation technique with low-complexity signal processing in frequency-domain to combat the multipath fading. The multiuser version of OFDM, namely orthogonal frequency-division multiple-access (OFDMA) and single-carrier FDMA, inherited its benefits and have be adopted in downlink and uplink, respectively, in the current fourth generation (4G) networks and they are playing the important role in wireless broadband communications [2]. For future fifth generation (5G) networks, coded-domain non-orthogonal multiple access (NOMA) scheme [3] has widely been recognized as a promising candidate owing to inheriting the features of spread spectrum multicarrier scheme including high spectrum efficiency and robustness against severe channel frequency selectivity. By using direct-sequence spreading, multicarrier direct-sequence code division multiple-access (MC DS-CDMA) can utilize very small number of subcarriers to accommodate a large number of users [4]. In addition to mitigating the peak-to-average power ratio (PAPR) problem, MC DS-CDMA also employs the flexibility to choose the relative values for the number of subcarriers and DS spreading factor, so that a good balance can be attained between the number of subcarriers and the DS spreading factor. With the above-mentioned merits, MC DS-CDMA may constitute one of the promising candidates for supporting multiuser communications in the future generations wireless mobile communications.

By contrast, SC-FDMA and OFDMA conveniently facilitate near-instantaneous adaptive subcarrier allocation for multiuser/relay assignment and scheduling by exploiting the knowledge of channel-state information of subcarriers [5, 6]. In [5], two dynamic resource allocation schemes are proposed by jointly considering subcarrier allocation with multiuser assignment and potential relay selection on the awareness of dual-hop channel quality and buffering delay criteria in order to improve the energy efficiency of SC-FDMA uplink. Our previous work in [6] designed two subcarrier allocation algorithms for OFDMA downlink by either avoiding the worst subchannel or seeking the best subchannel for user assignment, in order to trade-off between error performance and the spectrum efficiency. In order to find a good trade-off among spectral-efficient, high reliability and low complexity, intelligent dynamic resource allocation is desired by future multicarrier spread spectrum systems. However, in multicell multicarrier systems, severe intercell interference (InterCl) imposes a big challenge to resource management, where the interplay between InterCl and resource allocation should be addressed. In recent years, resource allocation in multicell multicarrier systems has drawn intensive research attention, as evidenced, e.g., by the references $[7,8,9,10,11,12,13,14]$ and references there 
in. In the literature, the studies of $[7,8,9,10]$, have investigated the centralized resource allocation in the multicell multicarrier systems. For example, the authors in [7, 9] have proposed the centralized approach by assuming base station (BS) cooperation. However, centralized resource allocation requires high implementation complexity and also consumes a big amount of backhaul resource. By contrast, distributed resource allocation demands relatively low implementation complexity, and has the capability of fast response to the dynamics of radio resources and wireless channels. Whereas, very limited literatures have barely studied distributed approaches for multicell multicarrier systems, e.g., in [11, 12, 13], most of which have been focused on OFDM systems.

In the literature, there are only very limited resource allocation studies for the MC DS-CDMA networks, such as $[15,16,17,18,19,20,21]$. In [15], the authors have proposed a novel framework for resource allocation in the context of the cognitive radio networks which employ the MC DS-CDMA technique. Considering the single-cell MC DS-CDMA systems, the authors in [19] have investigated the subcarrier-allocation and code-allocation, in which the spreading codes are not orthogonal. In $[16,17,18]$, the authors have proposed the code assignment schemes which can only deal with IntraCl. More recently, the resource allocation in the multicell downlink MC DS-CDMA systems has been studied in [20,21]. Specifically, in [20], motivating to maximizing the system sum rate, a novel resource allocation scheme has been proposed, in which the bisection approach is employed to iteratively solve the problem. The adaptive algorithm in [21] has been designed to allocate subchannel power and alphabet size in order to maximize the capacity of the multicell downlink MC DS-CDMA systems.

\section{2 | Motivations and Contributions}

To the best of our knowledge, there are no published references that have studied the allocation of spreading code (simply, code) in the multicell MC DS-CDMA systems. Against the background, we therefore motivate to investigate the resource allocation in the multicell downlink MC DS-CDMA systems, both subcarrier- and code-allocation are addressed. Future wireless communications, such that, $5 \mathrm{G}$ networks will have more stringent QoS requirements and link reliability requirements than fourth generation (4G) ones and will feature innovative strategies [22]. Hence, our resource allocation in the multicell MC DS-CDMA systems are designed with the objective to maximize the system's reliability (or minimize the average error probability).

The main contributions of this paper are summarized below.

- Motivated to achieving the high-reliability and low complexity, a general theoretical resource allocation framework is developed for the multicell downlink MC DS-CDMA systems. Our analysis shows that, the subcarrier- and code-allocation should be carried out separately, in order to reduce the implementation complexity, and robust to dynamics, as well as flexible for implementation without consuming unreasonable backhaul resources. Firstly, each BS independently carries out the subcarrier-allocation in its cell, with the objective to maximize the multiuser diversity. Then, code-allocation is operated based on the subcarrier-allocation results, with the objective to minimize the impact of InterCl.

- The novel resource allocation algorithms, namely simplified heuristic subcarrier- and code-allocation (SHSC), is proposed for the multicell downlink MC DS-CDMA systems. The SHSC is designed to minimize the implementation complexity while guaranteeing a relatively high reliability performance. the SHSC algorithm requires the BSs to exchange a minimum amount of the InterCl information about the slow fading (the InterCl factors). Furthermore, the characteristic analysis is carried out for the SHSC algorithm.

- We also propose another algorithm, namely the enhanced heuristic subcarrier- and code-allocation (EHSC), for the multicell downlink MC DS-CDMA systems. In comparison with the SHSC, the EHSC algorithm can obtain better sub- 
optimum solution to the decoupled mixed integer nonconvex optimization problem, while higher implementation complexity and more InterCl information are required. Both the SHSC and EHSC belong to the semi-distributed resource allocation approach, and they are capable of achieving a good trade-off between the reliability performance and the implementation complexity. Furthermore, the optimal subcarrier- and code-allocation (OSC) algorithm is also obtained to validate the performance of the proposed SHSC and EHSC algorithms.

- A range of performance analysis is provided and, specifically, the bit error rate (BER) performance of the proposed SHSC and EHSC algorithms are investigated and compared with a range of the existing algorithms and the OSC algorithm. The performance results demonstrate that the SHSC and EHSC algorithms are capable of outperforming the other existing sub-optimum algorithms and approaching the OSC. The performance results also reveal that the SHSC and EHSC can efficiently mitigate InterCI via code-allocation. Moreover, our simulation results demonstrate that the proposed algorithms do not make the performance trade-off between BER and spectral-efficiency.

The rest of the paper is organized as follows. Section 2 discusses the system model conceived. Section 3 analyzes the general theory of the resource allocation. Sections 4 and 5 detail the SHSC and EHSC algorithms, respectively. Section 6 provides the performance results and, finally, conclusions are summarized in Section 7.

\section{2 | SYSTEM MODEL}

Without loss of generality, the three-cell system model, such as [23, 24], is considered in order to catch the main features of multicell systems but make the problem relatively easy to manage. In the considered multicell systems, there are $K$ mobile users which are uniformly distributed in each hexagonal cell. Further, each BS and each user are assumed to equipped with only one antenna for communication. In our multicell MC DS-CDMA system, signals transmitted from BSs to users are assumed to be the MC DS-CDMA signals generated by length- $N$ time domain orthogonal DS spreading codes and transmitted on $M$ orthogonal subcarriers. We assume that each cell supports $K=M N$ users and, hence, each user is allocated one subcarrier and one spreading code. Note that, in the case that one user is assigned multiple subcarriers or/and codes, the system can be modified to use our model by dividing one user into several ones of each assigned one subcarrier and one code. In each cell, a user is allocated a unique pair of subcarrier and code, so that IntraCl can be avoided.

Furthermore, in each cell we assume for simplicity the ideal power control as in $[25,26,27,28]$, in order to maintain the same average received power of one unit per user, when there is no subcarrier/code-allocation. We also assume that each BS knows the channel state information (CSI) of its $K M$ intracell downlink channels. A BS only transmits signals to its intracell users. For convenience of description, the users in different cells sharing the same subcarrier and code are referred to as the co-subcarrier-code users. Explicitly, the co-subcarrier-code users interfere with each other, generating intercell interference (InterCI). Specifically, we define an InterCI factor as $\alpha=\sqrt{\left(\frac{d_{0}}{d_{1}}\right)^{\mu} 10^{\frac{\zeta_{0}-\zeta_{1}}{10}}}$, in which the large-scale fading including pathloss and shadowing can be evaluated under the power control [29]. Note that, $d_{0}$ and $d_{1}$ are the distances from the BS to the intracell user and the intercell user, respectively. We have the pathloss exponent denoted by $\mu$. Further, $\left(\zeta_{0}-\zeta_{1}\right)$ characterizes the shadowing effects of the InterCl, and it follows the log-normal distribution, which has the standard deviation $\Upsilon$ (in $\mathrm{dB}$ ) and zero mean. Moreover, in the multicell system, our downlink signals transmitted can be also affected by fast fading, which varies much more quickly (in particular may be hundreds of times quicker) than slow fading over the communication time. Without loss of generality, each BS is assumed to have the knowledge about channel state information (CSI) of its $K M$ intracell downlink channels. Further, for practical implementation, the BSs need to share the InterCl information for the co-subcarrier users only, in which 
TAB LE 1 Definitions of Key Notations.

\begin{tabular}{|c|r|c|c|}
\hline Notation & Definition & Notation & Definition \\
\hline$K$ & No. of Users & $M$ & No. of Subcarriers \\
\hline$N$ & No. of Codes & $\mathcal{M}$ & Set of Subcarrier Index \\
\hline$\alpha$ & InterCl factor & $\mathcal{N}$ & Set of Code Index \\
\hline$\gamma_{u, k}$ & SINR of User $k$ in Cell $u$ & $\mathcal{K}_{u}$ & Set of User Index in Cell $u$ \\
\hline$I_{u, k}$ & InterCI Power of User $k$ in Cell $u$ & $\boldsymbol{\Theta}_{m}^{\left(u, u^{\prime}\right)}$ & MInterCI Matrix \\
\hline $\mathcal{A}$ & Set of Subcarrier-Allocation Variables & $\mathcal{F}$ & Set of Subcarrier-Allocation Results \\
\hline $\mathcal{C}$ & Set of Code-Allocation Variables & $\mathcal{L}$ & Set of Code-Allocation Results \\
\hline$e_{k, k^{\prime}}$ & InterCI Score & $N_{b}$ & No. of MInterCI Bins \\
\hline
\end{tabular}

the required InterCl information is generated only by the slow fading, but not that due to fast fading ${ }^{1}$. As a result, this assumption for CSI imposes a small burden on feedback channels, and demands very low requirement of backhaul resources. To make this paper easy to follow, the key notations are summarized in Table 1.

Define that, $\mathcal{K}_{u}$ is the index set for users in cell $u$, while $\mathcal{M}$ is the index set for subcarriers in the system. Assume that, $\boldsymbol{x}_{u}=\left[x_{u, 0}, x_{u, 1}, \ldots, x_{u, K-1}\right]^{T}$ is the symbols desired by the $K$ users of cell $u$, in which $x_{u, k}$ is the symbol for user $k$. In general, for each $x_{u, k}$, it has zero mean and unit variance. The signals received by user $k$ can be given by

$$
\boldsymbol{r}_{u, k, m}=h_{u, k, m} \boldsymbol{Y}_{u}+\sum_{u^{\prime}=0, u^{\prime} \neq u}^{2} h_{u^{\prime}, k, m} \alpha_{u^{\prime}, k^{\prime}, k} \boldsymbol{Y}_{u^{\prime}}+\boldsymbol{n}_{u, k}
$$

where $k \in \mathcal{K}_{u}, m \in \mathcal{M}$, and assuming subcarrier $m$ is assigned to user $k . r_{u, k, m}$ is a length- $N$ vector observed by user $k$ of cell $u$. In the above equation, $h_{u, k, m}$ and $h_{u^{\prime}, k, m}$ are the fast fading channel gains for the intra-cell links and inter-cell links. While, $\alpha_{u^{\prime}, k^{\prime}, k}$ is the InterCl factor characterizing the slow fading of the InterCl channel between BS $u^{\prime}$ and user $k$, when user $k^{\prime}$ of cell $u^{\prime}$ is assumed on subcarrier $m$. The vector $\boldsymbol{n}_{u, k}$ for user $k$ is background noise, which follows the complex Gaussian distribution with a covariance matrix $2 \sigma^{2} \boldsymbol{I}_{N}$ and zero mean. Note that, $\bar{\gamma}_{s}$ is the average signal-to-noise ratio (SNR) per symbol, when there is no subcarrier/code allocation. In (1), $\boldsymbol{Y}_{u}=\boldsymbol{Q}_{m} \boldsymbol{G}_{u} \boldsymbol{x}_{u}$ is the transmit signal on subcarrier $m$ by BS $u$, and $\boldsymbol{Y}_{u^{\prime}}=\boldsymbol{Q}_{m} \boldsymbol{G}_{u^{\prime}} \boldsymbol{x}_{u^{\prime}}$ is the interference signal on subcarrier $m$ initiated from BS $u^{\prime}$.

Without loss of generality, in this paper we assume the orthogonal spreading codes $Q$ (size $(N \times N))$ are employed by the multicarrier systems. $\boldsymbol{Q}_{m}$ is a $(N \times K)$ matrix for the users on subcarrier $m$, and it is formed from matrix $Q$ by setting the columns corresponding to the subcarriers except $m$ to zero vectors. In addition to spreading the signals, a BS also need to pre-process the signal before transmission, such as, $\boldsymbol{G}_{u}=\operatorname{diag}\left\{\boldsymbol{g}_{u, 0}, \boldsymbol{g}_{u, 1}, \ldots, \boldsymbol{g}_{u, K-1}\right\}$ where $g_{u, k}=\left(h_{u, k, m}\right)^{*} / \sqrt{\left|h_{u, k, m}\right|^{2}}$. Observed from (1), the second term reflects the InterCI gain for user $k$. After applying the de-spreading on $\boldsymbol{r}_{u, k, m}$, we could obtain the decision variable, and deduce the signal-to-interference-plus-noise ratio

\footnotetext{
${ }^{1}$ As shown by the simulation results in Section 6, our performance improvements over the existing schemes are still significant. Our future work will investigate how the sharing of full InterCl information on both slow and fast fading among cells can help improving the resource allocation with acceptable complexity increased.
} 
(SINR) of user $k$ of subcarrier $m$, expressed as

$$
\gamma_{u, k, m}=\frac{\left|h_{u, k, m}\right|^{2}}{I_{u, k, m}+2 \sigma^{2}}
$$

where $k \in \mathcal{K}_{u}, m \in \mathcal{M}$. Note that, the InterCl gain $I_{u, k, m}$ can be given by

$$
I_{u, k, m}=\sum_{u^{\prime}=0, u^{\prime} \neq u}^{2}\left|h_{u^{\prime}, k, m} \alpha_{u^{\prime}, k^{\prime}, k} g_{u^{\prime}, k^{\prime}}\right|^{2}
$$

\section{3 | GENERAL THEORY}

In this section, we discuss the design objectives of our resource allocation including the subcarrier-and code-allocation for the multicell systems considered. Our resource allocation aims to minimize the average BER in order to design future high-reliability multicarrier spread spectrum systems. Correspondingly, our optimization problem can be given by

$$
\begin{gathered}
\min _{\mathcal{A}, C}\left\{\bar{P}_{e}=\frac{1}{3 K} \sum_{u=0}^{2} \sum_{k=0}^{K-1} \bar{P}_{e}^{(u, k)}\left(\gamma_{u, k}\right)\right\} \\
\text { subject to } \\
a_{u, k, m}=\{0,1\}, \forall m \in \mathcal{M}, \forall k \in \mathcal{K}_{u}, \forall u \in\{0,1,2\}, \\
c_{u, k, n}=\{0,1\}, \forall k \in \mathcal{K}_{u}, \forall n \in \mathcal{N}, \forall u \in\{0,1,2\}, \\
\sum_{m \in \mathcal{M}} a_{u, k, m}=1, \forall k \in \mathcal{K}_{u}, \forall u \in\{0,1,2\}, \\
\sum_{n \in \mathcal{N}} c_{u, k, n}=1, \forall k \in \mathcal{K}_{u}, \forall u \in\{0,1,2\}, \\
\sum_{k \in \mathcal{K}_{u}} a_{u, k, m}=N, \forall m \in \mathcal{M}, \forall u \in\{0,1,2\}, \\
\sum_{k \in \mathcal{K}_{u}} c_{u, k, n}=M, \forall n \in \mathcal{N}, \forall u \in\{0,1,2\} .
\end{gathered}
$$

In (4), $\bar{P}_{e}$ represents the average BER of the multicell system. $\bar{P}_{e}^{(u, k)}\left(\gamma_{u, k}\right)$ is the error probability of user $k$ of cell $u$, which is conditioned on the SINR $\gamma_{k}^{(u)}$ defined as follows.

$$
\gamma_{u, k}=\frac{\sum_{m \in \mathcal{M}} a_{u, k, m}\left|h_{u, k, m}\right|^{2}}{\sum_{u^{\prime}=0, u^{\prime} \neq u}^{2} I_{u, k}+2 \sigma^{2}}
$$

where

$$
I_{u, k}=\sum_{k^{\prime} \in \mathcal{K}^{\left(u^{\prime}\right)}} \sum_{m \in \mathcal{M}} \sum_{n \in \mathcal{N}} a_{u, k, m} a_{u^{\prime}, k^{\prime}, m} c_{u, k, n} c_{u^{\prime}, k^{\prime}, n}\left|h_{u^{\prime}, k, m} \alpha_{u^{\prime}, k^{\prime}, k} g_{u^{\prime}, k^{\prime}, m}\right|^{2}
$$


Above, $\mathcal{A}=\left\{a_{u, k, m}, \forall u, k, m\right\}$ and $C=\left\{c_{u, k, n}, \forall u, k, n\right\}$ are respectively the variable spaces for the subcarrier- and code-allocation. Specifically, $a_{u, k, m}=1$ if subcarrier $m$ is assigned to user $k$ of cell $u$, otherwise $a_{u, k, m}=0 . c_{u, k, n}=1$ if code $n$ is allocated to user $k$ of cell $u$, otherwise $c_{u, k, n}=0$. The constraints (7) and (9) require that, in each cell, one subcarrier is assigned to $N$ different users, and each user is allocated one subcarrier. For code-allocation, (8) and (10) impose the constraints that, in each cell, a user is assigned one code, and a code is allocated to $M$ different users. Note that, in the above equations $\mathcal{N}$ denotes the set of code indexes.

It is obviously known that, the optimization problem in (4) is a mixed integer nonlinear programming (MINLP) problem [30], which is nonconvex and very hard to solve. Moreover, to solve the problem (4), the BSs need to share the full InterCl information of all users, which is however not allowed according to our assumptions for the sake of practical implementation. Therefore, under the specific assumptions the optimization problem (4) can be decoupled into the problems (13) and (15), which indicate that our subcarrier- and code-allocation are carried out separately.

First of all, the subcarrier-allocation is carried out by each BS independently, since each BS is assumed to know the CSI of its intracell channels only. According to the results obtained in [31, 32, 33], minimizing the average system BER can be approximated by maximizing the SINR of each user, since $\bar{P}_{e}^{(u, k)}\left(\gamma_{u, k}\right)$ monotonically decreases as the SINR $\gamma_{u, k}$ increases. Hence, the subcarrier-allocation in each cell aims to maximize the numerator term of each user's SINR given in (11). This is equivalent to maximize the intracell subchannel qualities of each user, as done in [33], which can be expressed as

$$
\max _{\left\{\mathcal{A}_{u, m}, \forall m\right\}}\left\{\gamma_{u, k}^{\prime}, \forall k \in \mathcal{K}_{u}\right\}, \forall u \in\{0,1,2\}
$$

subject to (5), (7), (9)

where $\mathcal{A}_{u, m}=\left\{a_{u, k, m}, \forall k\right\}$ includes the subcarrier-allocation variables for subcarrier $m$ in cell $u$. In (13), the subchannel quality above can be reflected by the SNR of $\gamma_{u, k}^{\prime}$, given by

$$
\gamma_{u, k}^{\prime}=\sum_{m \in \mathcal{M}} \frac{a_{u, k, m}\left|h_{u, k, m}\right|^{2}}{2 \sigma^{2}}
$$

However, from (13), we can know that the problem for the subcarrier-allocation is still a mixed integer nonconvex problem.

As shown in (11), after the subcarrier-allocation, the users in different cells sharing the same subcarrier, which are referred to as the co-subcarrier users. They may interfere with each other, generating the InterCl. Therefore, after the subcarrier-allocation, we can carry out the code-allocation is to minimize the error probability of each co-subcarrier user group, which can be given as

$$
\min _{C_{m}}\left\{\frac{1}{3 N} \sum_{u=0}^{2} \sum_{k \in \mathcal{F}_{u, m}} \bar{P}_{e}^{(u, k)}\left(\gamma_{u, k} \mid\left\{\mathcal{A}_{m}^{(u)}, \forall u\right\}^{*}\right\}, \forall m \in \mathcal{M}\right.
$$

subject to (6), (8), (10).

In (15), the subcarrier-allocation results for subcarrier $m$ of cell $u$ is denoted by $\left\{\mathcal{A}_{u, m}\right\}^{*}$, which is equivalent to $\mathcal{F}_{m}^{(u)}$ which is index set of users allocated to subcarrier $m$ in cell $u$. However, the problem in (15) is still a mixed integer non-convex problem. It is still impossible to solve problem (15), since the BSs are assumed not to share the full InterCI information. 
Alternatively, our code-allocation can also be motivated to mitigate the InterCl for minimizing the error probability of each co-subcarrier user group, reflected by the summation part in the denominator of (11). Specifically, based on our assumption that BSs only share the InterCl information generated by slow fading, our code-allocation can only be carried out to minimize the InterCl factors of each co-subcarrier user group. Correspondingly, the optimization problem in (15) for the code-allocation becomes

$$
\min _{C_{m}}\left\{I_{u, k}, \forall k \in \mathcal{F}_{u, m}, \forall u \mid\left\{\mathcal{A}_{u, m}, \forall u\right\}^{*}\right\}, \forall m \in \mathcal{M}
$$

where

$$
\begin{aligned}
I_{k}^{(u)} & =\sum_{u^{\prime}=0, u^{\prime} \neq u}^{2} \sum_{k^{\prime} \in \mathcal{K}_{u^{\prime}}} \sum_{n \in \mathcal{N}} c_{u, k, n} c_{u^{\prime}, k^{\prime}, n}\left|\alpha_{u^{\prime}, k^{\prime}, k}\right|^{2} \\
& =\sum_{u^{\prime}=0, u^{\prime} \neq u}^{2} \tilde{I}_{k}^{u, u^{\prime}} .
\end{aligned}
$$

The problem in (16) is subject to (6), (8) and

$$
\sum_{k \in \mathcal{F}_{u, m}} c_{u, k, n}=1, \forall n \in \mathcal{N}, \forall u \in\{0,1,2\} .
$$

The constraint in (18) imposes that, after the subcarrier-allocation, only one code is assigned to each of the users in a co-subcarrier user group.

The code-allocation problem in (16) is a MINLP problem, which is NP hard to solve. Therefore, as done in [31, 32, 34], instead of solving the problem of (16), we employ the min-max approach to minimize the largest InterCl factor in each of the co-subcarrier user groups, which is described as

$$
\min _{\mathcal{C}_{m}}\left\{\max _{k \in \mathcal{F}_{u, m}, u}\left\{\tilde{I}_{k, \max }^{(u)}\right\} \mid\left\{\mathcal{A}_{u, m}, \forall u\right\}^{*}\right\}, \forall m \in \mathcal{M}
$$

where

$$
\begin{aligned}
& \tilde{I}_{k, \max }^{(u)}=\max _{u^{\prime} \neq u, u^{\prime} \in\{0,1,2\}}\left\{\tilde{I}_{k}^{u, u^{\prime}}\right\} \\
& \text { subject to (6), (8), (18). }
\end{aligned}
$$

As known, it requires much lower complexity to solve problems (13) and (19) than to solve the original problem of (4). However, the exhaustive search method is still needed.

\subsection{Optimal subcarrier- and code-allocation (OSC)}

As analyzed in [32], the authors have proved that the Hungarian algorithm can find the optimal solution to the problem same as (13) in the context of the single-cell multicarrier systems. Therefore, for the multicell MC DS-CDMA system, the optimal solution for the distributed subcarrier-allocation can be obtained by employing the Hungarian algorithm [35] independently at each BS. Furthermore, exhaustive search approach can be used to find the optimal solution to the mixed integer nonconvex problem (16). Therefore, in the context of the OSC algorithm, it first carries out the Hungarian 
algorithm based distributed subcarrier-allocation and, then, operates the exhaustive search based code-allocation. However, both the Hungarian algorithm and exhaustive search method require extremely high complexity, especially when the system has a large number of users, which prevents the OSC algorithm from practical implementation.

By contrast, in this paper we focus on designing practical meaningful low-complexity resource allocation algorithms, which motivate to find the sub-optimum solutions to the problems of (13) and (16) (or (19)). Correspondingly, two resource allocation algorithms including subcarrier- and code-allocation are proposed, which are the simplified heuristic subcarrier- and code-allocation (SHSC) and enhanced heuristic subcarrier- and code-allocation (EHSC) algorithms.

\section{4 | SIMPLIFIED HEURISTIC SUBCARRIER- AND CODE-ALLOCATION (SHSC) ALGORITHM}

In this section, we propose a novel resource allocation algorithm, namely SHSC. Under the SHSC, the subcarrierallocation is first operated by each BS independently. Then, the code-allocation is jointly carried out by two neighboring BSs, which is followed by the allocation at the other BS. The SHSC algorithm requires the BSs to know the intracell CSI for the subcarrier-allocation, and to exchange a part of the InterCl information about the slow fading (the InterCI factors) for the code-allocation.

\section{1 | Subcarrier-allocation}

In the multicell systems considered, the SHSC's subcarrier-allocation motivates to maximize the intracell subchannel qualities in each cell, as indicated by (13). As discussed in our paper [33], the worst case first (WCF) algorithm proposed for subcarrier-allocation is able to provide a good sub-optimum solution to the problem similar to (13). Hence, the SHSC algorithm employs the WCF algorithm with very low complexity required for the distributed subcarrier-allocation in our multicell systems.

As shown in [25, 32], the optimal solution to the problem similar to (13) can be obtained by the Hungarian algorithm, when the objective is to maximize the SNR of all users in the single-cell OFDMA systems employing the channel-inverse power-allocation. Furthermore, as shown in [33], the Hungarian algorithm can approximately solve the problem of (13). Therefore, for the sake of performance comparison, the benchmark of distributed subcarrier-allocation is thought of as the Hungarian algorithm [35] independently employed by each BS. However, the Hungarian algorithm also requires very high complexity, especially when the system has a large number of users, which becomes a bottleneck for practical applications.

In contrast to the Hungarian algorithm, the WCF subcarrier-allocation algorithm employed by the SHSC has much lower complexity and can be more easily implemented in practice. The basic principle of the WCF algorithm is to maximize the number of the worst intracell subchannel qualities which can be avoided. In a little more detail, the WCF algorithm first selects one of the allocation modes including user- and subcarrier-oriented, depending on which mode achieves higher diversity order. Then, it dynamically identifies the allocation order in an ascending way according to the worst subchannel qualities of the remaining users requiring allocation. Based on the order derived, the WCF algorithm always allocates the best available subchannel during each allocation iteration. With the aid of the dynamic ordering, more of the worst subchannels can be avoided assigning to users in comparison with the worst subcarrier avoiding (WSA) algorithm [32] which is one of the best sub-optimum subcarrier-allocation algorithms in literature. Consequently, the WCF algorithm can obtain a lower average error rate than the WSA, in addition to capturing all the advantages of the WSA algorithm. 
TAB LE 2 The InterCl factors $\left(\alpha_{u^{\prime}, k^{\prime}, k}, \alpha_{u, k, k^{\prime}}\right)$ between co-subcarrier users in cells 0 and 1.

\begin{tabular}{|c|c|c|c|c|}
\hline users & 18 & 21 & 22 & 26 \\
\hline 0 & $0.018,0.005$ & $0.108,0.003$ & $0.117,0.106$ & $0.143,0.006$ \\
\hline 3 & $0.067,0.126$ & $0.01,0.131$ & $0.026,1.958$ & $0.116,1.199$ \\
\hline 6 & $0.038,0.165$ & $0.011,0.137$ & $0.194,0.368$ & $0.147,0.205$ \\
\hline 7 & $0.065,0.022$ & $0.167,0.083$ & $0.019,0.177$ & $0.081,0.571$ \\
\hline
\end{tabular}

\section{2 | Code-allocation}

In the context of the SHSC algorithm, the code-allocation is operated after carrying out the WCF aided distributed subcarrier-allocation. Motivating to find promising sub-optimum solution to problem (19), the code-allocation should be designed to mitigate InterCl with very low complexity involved. Once the subcarrier-allocation is done, known by (17), the potential InterCI for each user only come from its co-subcarrier users. In that case, the SHSC operates the code-allocation independently for each co-subcarrier user group, based on the the InterCl information about slow fading (the InterCl factors) for each group is shared among the BSs. Therefore, we propose to minimize each InterCl of every single user by minimizing the maximum InterCl factor of each co-subcarrier user group.

In order to minimize the implementation complexity and backhaul burden, the code-allocation algorithm by the SHSC first allocates codes to the users in any two neighboring cells, then assigns codes to the users in the third cell. In the context of the code-allocation by the SHSC, there are $(U-1) N$ number of allocation iterations, in which $U=3$ is the number of cells. During each allocation iteration. the algorithm aims to assign a code to a pair of users or a group of users, so that the minimum available InterCl factors are identified. Without loss of generality, we can explain the principles of the code-allocation algorithms by using a typical example. Specifically, in the example, there are $M=4$ subcarriers and $N=4$ codes used to support $K=16$ users in each of the three cells and, hence, there are $3 N=12$ users on each subcarrier. Let us focus on the code-allocation for a typical subcarrier, saying subcarrier $m$. Assume that, the subcarrier-allocation results for subcarrier $m$ are: $\mathcal{F}_{0, m}=\{0,3,6,7\}$ for cell $0, \mathcal{F}_{1, m}=\{18,21,22,26\}$ in cell 1 , and $\mathcal{F}_{2, m}=\{32,33,36,41\}$ for cell 2. Correspondingly, we have the InterCl factors given by Tables 2-4 for the example considered. In each table, it gives the InterCI factors between each row and column user. For instance, in Table 2 , the elements $\left(\alpha_{u^{\prime}, k^{\prime}, k}, \alpha_{u, k, k^{\prime}}\right)$ represent the InterCl factor of the row-indexed user $k$ of cell $u$ caused by the column-indexed user $k^{\prime}$ of cell $u^{\prime}$, and the InterCl factor of the column-indexed user $k^{\prime}$ of cell $u^{\prime}$ imposed by the row-indexed user $k$ of cell $u$, respectively.

TAB LE 3 The InterCl factors $\left(\alpha_{u^{\prime}, k^{\prime}, k}, \alpha_{u, k, k^{\prime}}\right)$ between co-subcarrier users in cells 0 and 2.

\begin{tabular}{|c|c|c|c|c|}
\hline users & 32 & 33 & 36 & 41 \\
\hline 0 & $0.136,0.004$ & $0.624,0.002$ & $0.049,0.007$ & $0.218,0.008$ \\
\hline 3 & $0.054,0.031$ & $0.102,0.101$ & $0.012,0.146$ & $0.064,0.201$ \\
\hline 6 & $0.167,0.033$ & $0.013,0.14$ & $0.228,0.464$ & $0.13,0.019$ \\
\hline 7 & $2.243,0.034$ & $0.656,0.127$ & $0.089,0.058$ & $0.063,0.139$ \\
\hline
\end{tabular}

Based on the example in Tables 2-4, the principles of the code-allocation by the SHSC can be explained as follows. For code-allocation, let us first introduce the modified InterCI (MInterCl) matrices $\Theta_{m}^{(0,1)}, \Theta_{m}^{(0,2)}$ and $\Theta_{m}^{(1,2)}$. Each element 
TAB LE 4 The InterCl factors $\left(\alpha_{u^{\prime}, k^{\prime}, k}, \alpha_{u, k, k^{\prime}}\right)$ between co-subcarrier users in cells 1 and 2.

\begin{tabular}{|c|c|c|c|c|}
\hline users & 32 & 33 & 36 & 41 \\
\hline 18 & $0.095,0.052$ & $0.057,0.125$ & $0.04,0.23$ & $0.098,0.017$ \\
\hline 21 & $0.129,0.229$ & $0.215,0.024$ & $1.127,0.166$ & $0.144,0.044$ \\
\hline 22 & $0.009,0.045$ & $0.096,0.912$ & $0.077,0.47$ & $0.011,0.133$ \\
\hline 26 & $0.245,0.014$ & $0.729,0.015$ & $0.18,0.623$ & $0.041,0.068$ \\
\hline
\end{tabular}

in an MInterCl matrix is obtained from the InterCl factors in the corresponding Table (including Tables 2 -4). For example, element $\tilde{\alpha}_{k, k^{\prime}}^{\left(u, u^{\prime}\right)}$ in $\Theta_{m}^{\left(u, u^{\prime}\right)}$ can be derived by

$$
\tilde{\alpha}_{k, k^{\prime}}^{\left(u, u^{\prime}\right)}=\max \left\{\alpha_{u^{\prime}, k^{\prime}, k}, \alpha_{u, k, k^{\prime}}\right\}, \quad k \in \mathcal{F}_{u, m}, k^{\prime} \in \mathcal{F}_{u^{\prime}, m}
$$

in which the larger InterCI factor is kept, while the smaller one is discard. In that case, we have MInterCl matrix $\Theta_{m}^{(0,1)}$ given by

$$
\Theta_{m}^{(0,1)}=\left[\begin{array}{ccccc} 
& U_{18} & U_{21} & U_{22} & U_{26} \\
U_{0} & 0.018 & 0.108 & \underline{0.117} & 0.143 \\
U_{3} & \underline{0.126} & 0.131 & 1.958 & 1.199 \\
U_{6} & 0.165 & 0.137 & 0.368 & \underline{0.205} \\
U_{7} & 0.065 & \underline{0.167} & 0.177 & 0.571
\end{array}\right] \text { (2) }
$$

Similarly, we could derive MInterCl matrices $\Theta_{m}^{(0,2)}$ and $\Theta_{m}^{(1,2)}$, which are omitted due to the lack of space.

We are now able to implement the code-allocation based on the three $\mathrm{MInterCl}$ matrices. The code-allocation is first carried out for the users in cells 0 and 1 with the aid of $\Theta_{m}^{(0,1)}$, then is operated for the users in cell 0 with the help of $\Theta_{m}^{(1,2)}$. Note that, the code-allocation order for the three cells does not affect the performance of the proposed algorithm. As shown in (22), the circled numbers denote the indices of code-allocation iterations, which will be explained in the following. The underlined numbers in the matrix indicate the allocation result, corresponding to the pairs of co-code users which are assigned the same code. As shown in (2) and (3), when the InterCl factor is higher than 1, i.e., when $\alpha_{u^{\prime}, k^{\prime}, k}>1$, the InterCl imposes severe effect on the detection of the desired signal. Therefore, let us define that, the users generating the InterCl factors $\left\{\tilde{\alpha}_{k, k^{\prime}}^{\left(u, u^{\prime}\right)}>1\right\}$ in the $\mathrm{MInterCl}$ matrix as the undesirable users. Furthermore, during the code-allocation, a user becomes a unavailable user if the user has been allocated a code. During each allocation iteration, we first identify the row user which has the maximum number of the co-subcarrier column users being undesirable or unavailable. When considering $\Theta_{m}^{(0,1)}$, in iteration 1 , we find that user 3 is the one having the most undesirable users, including user 22, user 26 of cell 1 , since $\tilde{\alpha}_{3,22}^{(0,1)}=1.958>1$ and $\tilde{\alpha}_{3,26}^{(0,1)}=1.199>1$. Then, we search the minimum MInterCl factor for the user identified. For user 3 , we have the minimum one 0.126 , which corresponding to the InterCl from user 18 in cell 1 . As a result, we find a pair of co-code users, i.e. user 3 and user 18 , which is denoted by the underlined element. Since there is no code assigned, the SHSC algorithm allocates code 0 to user 3 and user 18. Known from (22), after iteration 1, each remaining user has the only one unavailable user, which is user 18 . In order to avoid strong potential InterCl, a code is assigned to the user with the maximum MInterCl factor. Observed from (22), we know that the maximum MInterCl factor is $\tilde{\alpha}_{7,26}^{(0,1)}=0.571$ and, hence, user 7 and user 26 are identified as a pair of co-code users. Therefore, we assign code 1 to user 21 in cell 1 as the co-code user of user 7 in cell 0 . In iteration 3 and 4 , we assign codes 
to the remaining users in a similar approach. As a result, the code-allocation for cell 0 and cell 1 is completed, and the the underlined elements indicate the assignment.

Let us now proceed to the code-allocation for cell 2 . We should note that, we can now carry out the allocation process by using $\Theta_{m}^{(0,2)}$ or $\Theta_{m}^{(1,2)}$, which may give a different allocation results for each time but can achieve the same average BER performance. Without loss of generality, the code-allocation for cell 2 is described by using matrix $\boldsymbol{\Theta}_{m}^{(0,2)}$, given by

$$
\Theta_{m}^{(0,2)}=\left[\begin{array}{ccccc} 
& U_{32} & U_{33} & U_{36} & U_{41} \\
U_{0} & \underline{0.136} & 0.624 \rightarrow 0.912 & 0.049 \rightarrow 0.47 & 0.218 \\
U_{3} & 0.054 \rightarrow 0.095 & \underline{0.102 \rightarrow 0.125} & 0.146 \rightarrow 0.23 & 0.201 \\
U_{6} & 0.167 \rightarrow 0.245 & 0.14 \rightarrow 0.729 & \underline{0.464 \rightarrow 0.623} & 0.13 \\
U_{7} & 2.243 & 0.656 & 0.089 \rightarrow 1.127 & \underline{0.139 \rightarrow 0.144}
\end{array}\right] \text { (6) }
$$

Before allocation, we need to update the values in $\Theta_{m}^{(0,2)}$, since the users in cell 0 have been allocated codes. In (23), ' $a \rightarrow b$ ' represents ' $a$ ' is replaced by ' $b$ ' since ' $b>a$ ', where ' $a$ ' is the MInterCl factor before code-allocation. Therefore, $\Theta_{m}^{(0,2)}$ becomes

$$
\tilde{\alpha}_{k, k^{\prime \prime}}^{\left(u, u^{\prime \prime}\right)}=\max \left\{\tilde{\alpha}_{k, k^{\prime \prime}}^{\left(u, u^{\prime \prime}\right)}, \tilde{\alpha}_{k^{\prime}, k^{\prime \prime}}^{\left(u, u^{\prime \prime}\right)}\right\}, k \in \mathcal{L}_{0, n}, k^{\prime} \in \mathcal{L}_{1, n}, k^{\prime \prime} \in \mathcal{F}_{2,0}
$$

where $\mathcal{L}_{n}^{(u)}$ is the index set for users in cell $u$ which have been assigned code $n$.

Based on (23), the users in cell 2 sharing subcarrier $m$ are allocated the codes as follows. In detail, during the 5 th iteration, user 7 in cell 0 is identified, owing to it has the most number of undesirable users, which is known by the fact that $\tilde{\alpha}_{7,32}^{(0,2)}=2.243$ and $\tilde{\alpha}_{7,36}^{(0,2)}=1.127$. Therefore, user 41 and user 7 are identified as a pair of co-code users, since the minimum average InterCl factor $\tilde{\alpha}_{7,41}^{(0,2)}=0.144$ is available. Known from allocation iteration 2, code 1 has been given to user 7 . Hence, user 41 is also allocated code 1 . Similarly, the code-allocation can be implemented for the other three users in cell 2 during iteration 6 - 8. In summary, when considering the above example, the code-allocation results for the co-subcarrier users are denoted by the underlines in (22) and (23). Therefore, the SHSC algorithm derives the maximum InterCI factor $\alpha^{(\max )}=\alpha_{1,26,36}=0.623$, and the average InterCI factor $\alpha^{(\text {ave })}=0.145$. The principles of the SHSC's code-allocation can be summarized below.

In summary, can be stated as follows.

\section{Code-Allocation of the SHSC algorithm:}

For subcarrier $m=0,1, \ldots, M-1$ :

Initialization:

Set $\mathcal{L}_{u, n}=\varnothing, \forall n \in \mathcal{N}, \forall u \in\{0,1,2\}$;

Set $u \neq u^{\prime} \neq u^{\prime \prime}, u, u^{\prime}, u^{\prime \prime} \in\{0,1,2\}$;

Form MInterCl matrices $\Theta_{m}^{\left(u, u^{\prime}\right)}, \forall u, u^{\prime}$, according to (21);

Set $\tilde{\mathcal{K}}_{u, s}=\varnothing, \forall s=1,2, \ldots, 2 N ; \tilde{\mathcal{N}}=\mathcal{N}, \tilde{\mathcal{F}}_{u, m}=\mathcal{F}_{u, m}, \tilde{\mathcal{F}}_{u^{\prime}, m}=\mathcal{F}_{u^{\prime}, m}, \forall u, u^{\prime} ;$

For iteration $s=1, \ldots, 2 N$, execute:

Step 1 User identification:

(a) Identify the user/users in cell $u$ having the maximum number of unavailable plus undesirable users from cell $u^{\prime}$. Let $\tilde{\mathcal{K}}_{u, s}$ contain the identified user/users indexes.

(b) Find the user in $\tilde{\mathcal{K}}_{u, s}$, which has the largest MInterCl factor: 


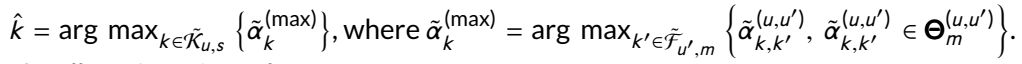

Step 2 Allocation of a code:

Allocate code $n$ to users $\hat{k}$ and $\hat{k}^{\prime}: \mathcal{L}_{u, n}=\{\hat{k}\}, \mathcal{L}_{u^{\prime}, n}=\left\{\hat{k}^{\prime}\right\}$, where $\hat{k}^{\prime}=\arg \min _{k^{\prime} \in \tilde{\mathcal{F}}_{u^{\prime}, m}}\left\{\tilde{\alpha}_{\hat{k}, k^{\prime}}^{\left(u, u^{\prime}\right)}, \tilde{\alpha}_{k, k^{\prime}}^{\left(u, u^{\prime}\right)} \in \boldsymbol{\Theta}_{m}^{\left(u, u^{\prime}\right)}\right\}$. $n=j$ if $\hat{k} \in \mathcal{L}_{u, j}$ or $\hat{k}^{\prime} \in \mathcal{L}_{u^{\prime}, j}$; otherwise $n=\tilde{\mathcal{N}}(0)$.

Step 3 Update:

Update $\Theta_{m}^{\left(u, u^{\prime}\right)}$ by (24), if $s \leq N$; Set $\tilde{\mathcal{F}}_{u, m} \leftarrow \tilde{\mathcal{F}}_{u, m}-\{\hat{k}\}, \tilde{\mathcal{F}}_{u^{\prime}, m} \leftarrow \tilde{\mathcal{F}}_{u^{\prime}, m}-\left\{\hat{k}^{\prime}\right\}$; Set $\tilde{\mathcal{N}} \leftarrow \tilde{\mathcal{N}}-n$; Set $u^{\prime}=u^{\prime \prime}$ if $s=N$. Step 4 Re-do Steps $1-3$ until $\tilde{\mathcal{N}}=\varnothing$.

As shown in Algorithm 1, the SHSC algorithm completes the code-allocation in $2 N$ iterations. In order to achieve low implementation complexity, firstly, the SHSC's code-allocation jointly assigns the codes to the users in two neighboring BSs based on the InterCI factors exchanged between these two BSs. Then, the third BS assigns codes to its users based on the allocation results for the other two cells. Therefore, the code-allocation proposed in the SHSC algorithm is actually a semi-centralized approach, which requires the BSs to exchange a relatively small amount of InterCl information, in comparison to the code-allocation under the EHSC algorithm in the next section.

\section{5 | ENHANCED HEURISTIC SUBCARRIER- AND CODE-ALLOCATION (EHSC) ALGORITHM}

In this section, we propose another novel resource allocation algorithm called EHSC. In comparison with the SHSC, the EHSC aims to find better sub-optimum solutions to the optimization problems of (13) and (19) with a reasonable amount of complexity increased. Under the EHSC algorithm, the BSs first carry out the distributed subcarrier-allocation, then they jointly operate the code-allocation. Furthermore, the EHSC algorithm requires the BSs to know their intracell $\mathrm{CSI}$, and to share the InterCl information generated by the slow fading.

\section{1 | Subcarrier-allocation}

The EHSC employs the iterative worst excluding (IWE) aided WCF algorithm referring to the IWE-WCF [33] for the distributed subcarrier-allocation. In comparison with the WCF algorithm employed by the SHSC, the IWE-WCF subcarrier-allocation algorithm can achieve a better BER performance but requires a slight higher implementation complexity.

The IWE-WCF algorithm proposed in our paper [33] can iteratively update the associated subchannel quality matrix during subcarrier-allocation. In a little detail, during an iteration the algorithm first deletes the candidate subchannels corresponding to the worst unassigned channel qualities, then the subcarrier-allocation is completed by the WCF algorithm. At the end of each iteration, the allocation result is compared with that obtained from the last iteration. If performance gain is observed, then the subcarrier-allocation is executed again. This procedure stops, when there is no further performance gain or when subcarrier-allocation cannot be accomplished. As shown in [33], the IWE algorithm can be also easily integrated with the WSA, WCA algorithms, forming the IWE-WSA, IWE-WCA algorithms, respectively. Our studies show that the IWE assisted algorithms always outperform the original algorithms without using IWE. Furthermore, an IWE-aided algorithm is low-complexity for operation, and it can usually complete the allocation after a low number, typically $1-3$, of iterations. 


$$
\Theta_{m}^{(0,1)}=\left[\begin{array}{ccccc} 
& U_{18} & U_{21} & U_{22} & U_{26} \\
U_{0} & 0.018 & 0.108 \rightarrow 0.229 & 0.117 & 0.143 \rightarrow 0.245 \\
U_{3} & \underline{0.126} & 0.131 & 1.958 & 1.199 \\
U_{6} & 0.165 & \underline{0.137 \rightarrow 0.215} & 0.368 \rightarrow 0.912 & 0.205 \rightarrow 0.729 \\
U_{7} & 0.065 & 0.167 & \underline{0.177} & 0.571
\end{array}\right]
$$

(8)

$$
\Theta_{m}^{(0,2)}=\left[\begin{array}{ccccc} 
& U_{32} & U_{33} & U_{36} & U_{41} \\
U_{0} & \underline{0.136} & 0.624 & 0.049 & 0.218 \\
U_{3} & 0.054 \rightarrow 0.095 & 0.102 \rightarrow 0.125 & \underline{0.146 \rightarrow 0.23} & 0.201 \\
U_{6} & 0.167 & \underline{0.14} & 0.464 & 0.13 \\
U_{7} & 2.243 & 0.656 & 0.089 & \underline{0.139}
\end{array}\right] \text { (5) }
$$

\section{2 | Code-allocation}

The code-allocation of the SHSC requires very low complexity, as it only considers two $(N \times N)$ InterCl matrices at a time. Interestingly, with a slight increase of complexity, the error rate performance of the multicell system can be improved. In the SHSC algorithm, the code-allocation algorithm motivates to avoid the strong InterCl generated by two neighboring cells at each time, while ignoring the InterCl effect imposed by the other cell in the context of the three cell considered. Therefore, the performance of the code-allocation under the SHSC may be limited by the fact that, the code-allocation in last iterations may result in strong InterCI. With reasonable amount of complexity increased, our EHSC algorithm proposes another approach to carry out code-allocation. For this sake, it aims to minimize the maximum InterCl factor by jointly considering the three neighboring cells. The principles of the code-allocation of the EHSC can be described by using the same example in Tables 2-4. For the example considered, the allocation process can be explained by the MInterCl matrices in (25)-(27).

The main difference between the code-allocation algorithms under the SHSC and the EHSC is that the EHSC's code-allocation identifies a user having the maximum number of unavailable plus undesirable users, which is found from all the three MInterCl matrices in (25)-(27). As a result, it certainly achieves a higher selection diversity. However, the SHSC's code-allocation finds a user of this kind from just one MInterCl matrix, as shown in Section 4. In addition, to order to maximize the selection diversity, the code-allocation process operates the searching from both the row and column directions of the $\mathrm{MInterCl}$ matrices. 
The code-allocation in the EHSC algorithm, given by (25) - (27), demands eight iterations in total to accomplish the code-allocation. In the first iteration, user 3 in cell 0 is identified, since it has 2 undesirable users, corresponding to the maximum number of unavailable plus undesirable users. Then, we find that the smallest InterCl factor in the row is $\tilde{\alpha}_{3,18}=0.126$ and, hence, user 18 and user 3 are identified as a pair of co-code users, which can be assigned code 0 . With the aid of method introduced in (24), the information about the MInterCl factors need to be updated in $\Theta_{m}^{(0,2)}$ and $\Theta_{m}^{(1,2)}$. Shown by (26) and (27), it becomes $\tilde{\alpha}_{3, k^{\prime \prime}}^{\left(0, u^{\prime \prime}\right)}=\tilde{\alpha}_{18, k^{\prime \prime}}^{\left(1, u^{\prime \prime}\right)}, \forall k^{\prime \prime} \in \mathcal{F}_{2, m}$.

Proceeding to the second iteration, the code-allocation algorithm under the EHSC needs to find the user with the largest number of unavailable users plus undesirable users among the available options. Nevertheless, we find that, each of the remaining users has one undesirable user. In order to tackle this problem, we propose a new approach to obtain the user priority, namely the InterCI scoring (IS). The IS approach is designed to minimize the largest value of InterCl factor for users, and its principle can be given as follows.

InterCI Scoring (IS): Given a MInterCI factor $\tilde{\alpha}_{k, k^{\prime}}^{\left(u, u^{\prime}\right)}$, the corresponding InterCl score is computed by

$$
e_{k, k^{\prime}}=i, \text { if } \tilde{\alpha}_{k, k^{\prime}}^{\left(u, u^{\prime}\right)} \in \mathcal{B}_{i}, i=0,1, \ldots, N_{b}+1
$$

where $\mathcal{B}_{i}$ denotes the $i$ th $\mathrm{MInterCl}$ bin and there are in total $\left(N_{b}+2\right)$ number of $\mathrm{MInterCl}$ bins, and $\mathcal{B}_{i}$ is defined as

$$
\mathcal{B}_{i}= \begin{cases}{[1,+\infty),} & \text { if } i=0, \\ \left(1-0.9 i / N_{b}, 1-0.9(i-1) / N_{b}\right], & \text { if } i=1, \ldots, N_{b}, \\ (0,0.1), & \text { if } i=N_{b}+1 .\end{cases}
$$

According to (28) and (29), the IS method maps the MInterCI factors to different scores. In particular, a higher $\mathrm{MInterCl}$ factor value corresponds to a lower score, and the corresponding allocation is less desirable. As defined in (29), we can categorize the Inter $\mathrm{Cl}$ factors into different regions: 1 ) ignorable InterCl, scoring $N_{b}+1$ for $0<\tilde{\alpha}_{k, k^{\prime}}^{\left.\left(u, u^{\prime}\right)<0.1 ; 2\right)}$ moderate InterCl, scoring $e_{k, k^{\prime}} \in\left\{1, \ldots, N_{b}\right\}$ for $0.1 \leq \tilde{\alpha}_{k, k^{\prime}}^{\left(u, u^{\prime}\right)}<1$; 3) strong InterCl, scoring zero for $\tilde{\alpha}_{k, k^{\prime}}^{\left(u, u^{\prime}\right)} \geq 1$.

By leveraging the IS approach, we are now able to find the user having the highest priority for code-allocation during iteration 2. The user is identified by searching the lowest sum of IS scores, which corresponds to the strongest InterCI effect. For the example, when assuming $N_{b}+2=4$, user 36 in cell 2 is identified for allocating a code, due to the fact that, it has the lowest sum vaule of the IS scores being $e_{18,36}+e_{21,36}+e_{22,36}+e_{26,36}=5$. Given by (27), user 18 and user 36 are identified as a pair of co-code users, since $\tilde{\alpha}_{18,36}^{(1,2)}=0.23$ is the minimum MInterCI factor available. We note that, users 3 and 18 have been assigned code 0 in the first iteration. Therefore, by combining the result in iteration 2, we know that users 3,18 and 36 become the co-code users. Furthermore, indicated by the underlined element of $(26)$, the $\mathrm{MInterCl}$ factor $\tilde{\alpha}_{3,36}^{(0,2)}$ is automatically identified, since user 36 is a co-code user of user 3 . Note that, the rest code-allocation can be carried out in a similar approach as those done in the above iterations 1 and 2 . As a result, the final allocation results can be found in (25) - (27), and they are denoted by the underlined elements. The results can be given as: code 0 is assigned to users 3,18 and 36 , code 1 is given to users 7, 22 and 41, code 2 is allocated to users 0,26 and 32 sharing, and code 3 is for users 6, 21 and 33. In that case, we derive the maximum InterCl factor $\alpha^{(\max )}=\alpha_{2,32,26}=0.245$, and the average InterCI factor $\alpha^{\text {(ave.) }}=0.093$, which certainly outperforms the SHSC in terms of the average BER. In conclusion, the performance gain achieved by the EHSC over the SHSC is simply due to a higher selection diversity exploited but with higher implementation complexity required.

In general, the EHSC's code-allocation algorithm can be summarized as follows. Code-Allocation in the EHSC Algorithm:

For subcarrier $m=0,1, \ldots, M-1$ : 


\section{Initialization:}

Set $u \neq u^{\prime}$;

Set $\mathcal{L}_{u, n}=\varnothing, \forall n \in \mathcal{N}, \forall u \in\{0,1,2\}$;

Form the MInterCl matrices $\Theta_{m}^{\left(u, u^{\prime}\right)}, \forall u, u^{\prime} \in\{0,1,2\}$, according to (21);

Set $\tilde{\mathcal{K}}_{s}=\varnothing, \forall s=1,2, \ldots, 2 N ; \tilde{\mathcal{N}}=\mathcal{N}, \tilde{\mathcal{F}}_{u, m}=\mathcal{F}_{u, m}, \forall u \in\{0,1,2\}$.

For iteration $s=1, \ldots, 2 N$, execute:

Step 1 User identification:

(a) Identify the user/users related to a specific MInterCl matrix, which has/have the maximum number of the unavailable users plus undesirable users. Let $\tilde{\mathcal{K}}_{s}$ contain the identified user/users indexes.

(b) Identify user $\hat{k}$ in $\tilde{\mathcal{K}}_{s}$, which has the minimum sum of IS:

$\hat{k}=\arg \max _{k \in \tilde{\mathcal{K}}_{s}}\left\{E_{k}\right\}$, where $k \in \mathcal{F}_{u, m}, E_{k}=\sum_{k^{\prime} \in \tilde{\mathcal{F}}_{u^{\prime}, m}} e_{k, k^{\prime}}$ if $u<u^{\prime}$, or $E_{k}=\sum_{k^{\prime} \in \tilde{\mathcal{F}}_{u^{\prime}, m}} e_{k^{\prime}, k}$ if $u>u^{\prime}$.

Step 2 Allocation of a code:

Allocate code $n$ to users $\hat{k}$ and $\hat{k}^{\prime}: \mathcal{L}_{u, n}=\{\hat{k}\}, \mathcal{L}_{u^{\prime}, n}=\left\{\hat{k}^{\prime}\right\}$, where $n=j$ if $\hat{k} \in \mathcal{L}_{u, j}$ or $\hat{k}^{\prime} \in \mathcal{L}_{u^{\prime}, j}$; otherwise $n=\tilde{N}(0)$. $\hat{k}^{\prime}=\arg \min _{k^{\prime} \in \tilde{\mathcal{F}}_{u^{\prime}, m}}\left\{\tilde{\alpha}_{\hat{k}, k^{\prime}}^{\left(u, u^{\prime}\right)}, \tilde{\alpha}_{\hat{k}, k^{\prime}}^{\left(u, u^{\prime}\right)} \in \Theta_{m}^{\left(u, u^{\prime}\right)}\right\}$ if $u<u^{\prime} ;$

$\hat{k}^{\prime}=\arg \min _{k^{\prime} \in \tilde{\mathcal{F}}_{u^{\prime}, m}}\left\{\tilde{\alpha}_{k^{\prime}, \hat{k}}^{\left(u, u^{\prime}\right)}, \tilde{\alpha}_{\hat{k}, k^{\prime}}^{\left(u, u^{\prime}\right)} \in \boldsymbol{\Theta}_{m}^{\left(u, u^{\prime}\right)}\right\}$ if $u>u^{\prime}$.

Step 3 Update:

Update $\boldsymbol{\Theta}_{m}^{\left(u, u^{\prime}\right)}$ by $(24)$, set $\tilde{\mathcal{F}}_{u, m} \leftarrow \tilde{\mathcal{F}}_{u, m}-\{\hat{k}\}, \tilde{\mathcal{F}}_{u^{\prime}, m} \leftarrow \tilde{\mathcal{F}}_{u^{\prime}, m}-\left\{\hat{k}^{\prime}\right\}, \forall u, u^{\prime} \in\{0,1,2\} ;$ Set $\tilde{\mathcal{N}} \leftarrow \tilde{\mathcal{N}}-n$.

Step 4 Re-do Steps $1-3$ if $\tilde{\mathcal{N}} \neq \varnothing$.

As Algorithm 2 shows, the EHSC's code-allocation jointly assigns the codes to the users in the three cells. During an iteration of code-allocation in the EHSC algorithm, it identifies the three co-code users of a subcarrier by simultaneously considering all the three $\mathrm{MInterCI}$ matrices, instead of considering only one in the SHSC algorithm. Therefore, the EHSC will exploit a higher selection diversity than the SHSC for allocating spreading code. In the context of the multicell systems, the EHSC algorithm can attain a higher average SINR, therefore, it obtains a better system reliability and also a higher spectral-efficiency than the SHSC. However, the EHSC requires higher implementation complexity for code-allocation than the SHSC algorithm.

\section{3 | Characteristic Analysis}

For the multicell MC DS-CDMA systems considered, the design of the proposed resource allocation guarantees a relatively low complexity for practical implementation. Specifically, in our resource allocation, subcarrier-allocation is first implemented independently at each of the BSs with only the knowledge of intracell channel information. Then the InterCI mitigation is handled via code-allocation, which requires the BSs to share a part of users' channel information about only the propagation pathloss and shadowing. Furthermore, in our scheme, code-allocation is operated independently for the co-subcarrier user groups of each having $3 N$ users. By contrast, when the multicarrier system without using DS spreading, such as OFDMA, is employed, the number of subcarriers per cell may be as high as $M N$, when the same system bandwidth is considered. In this case, a resource allocation algorithm needs to simultaneously deal with $3 M N$ users in three cells, which may demand an extremely high complexity.

In comparison to the EHSC's code-allocation, the SHSC algorithm's code-allocation has a big advantage of demanding very low complexity since it carries out the allocation successively for each cell. Furthermore, the code-allocation proposed by the SHSC can be thought of as a semi-distributed approach, which requires the BSs to exchange a relatively small amount of InterCl information. By jointly considering the code-allocation of the three cells, the EHSC can exploit a higher selecting diversity than the SHSC algorithm. As a result, by contributing more complexity, the EHSC 
algorithm is capable of attaining a better performance in terms of InterCl mitigation for the multicell systems than the SHSC algorithm. When considering practical systems with more than three cells, our proposed SHSC and EHSC algorithms are still applicable. In that case, the SHSC and EHSC will first carry out their distributed subcarrier-allocation algorithms, which demand intracell CSI locally. Then, The worst InterCl scenario for a user happens in practical systems is that, a user seats close to edge of three cells. In that case, this user could suffer from strong InterCl caused by the transmissions in the two neighboring cells. Therefore, in the practical systems, we will divide the cells into different groups of each including three neighboring cells. For each cell group, the SHSC and EHSC algorithms independently implement the code-allocation based on the results of subcarrier-allocation. Note that, by using this approach, our proposed algorithms will cause very small amount of signaling burden on feedback channels, which is a big advantage for practical implementation.

Nevertheless, both the proposed SHSC and EHSC algorithms requires much less complexity than the OSC. Let us now discuss the complexity of the algorithms involved, which can be counted by the number of comparisons. In our analysis, we assume that, there are $M$ subcarriers and $N$ codes to support $3 K=3 M N$ users in the three-cell MC DS-CDMA system. The OSC algorithm, proposed in Section 3, employs the Hungarian algorithm for distributed subcarrier-allocation, which requires $\left(11 K^{3}+12 K^{2}+31 K\right) / 2$ comparisons for the multicell system [35]. Furthermore, the OSC uses the exhaustive search approach for code-allocation, which needs $(N !)^{3}$ for each co-subcarrier user group containing $3 N$ users. Therefore, the complexity of the OSC algorithm is given by $O\left(\max \left\{K^{3}, M(N !)^{3}\right\}\right)$.

In the context of the SHSC, the WCF algorithm for distributed subcarrier-allocation requires at most $(2 K-1)(3 M-$ $3)+\frac{3}{2} K(K-1)$ comparisons, which is known from [33]. Further, based on two MInterCl matrices, the SHSC algorithm requires $2 N$ iterations to operate the code-allocation independently for each co-subcarrier user group. As shown by Algorithm 1, the complexity required by the code-allocation process mainly includes finding unavailable users (requiring $\left(N^{2}-2 N+5\right)(N-2)$ comparisons), identifying the maximum MInterCl factors (requiring $\mathcal{K}_{s}^{(u)}$ comparisons), and allocation of the best MInterCl factors (requiring $2 N^{2} \ln N$ comparisons). In summary, we can conclude that, the complexity of the SHSC algorithm including both subcarrier- and code-allocation is $O\left(\max \left\{K^{2}, M N^{3}\right\}\right)$.

By contrast, our EHSC algorithm employs the IWE-WCF subcarrier-allocation algorithm, whose complexity has been analyzed in [33]. For each co-subcarrier user group, the EHSC's code-allocation operates the allocation by simultaneously considering all the three MInterCl matrices. The analysis for calculating the EHSC's code-allocation is similar to that for the SHSC's code-allocation, and this analysis process is omitted due to the lack of space. In total, the complexity of the EHSC algorithm is $O\left(\max \left\{S K^{2}, M N^{3}\right\}\right)$. From the above complexity analysis, we readily know that both the proposed SHSC and EHSC algorithms require significantly smaller amount of complexity than the optimal algorithm, i.e. the OSC. Furthermore, the low complexity of the SHSC and EHSC algorithms will be validated by comparing with the other low complexity algorithms in Section 6. Although our proposed algorithms demand low complexity, they may still cause noticeable delay in practical implementation, especially when the number of users in the system is relatively large. This is due to the fact that, it requires more signalling burden on the feedback channels and backhaul channels for large systems. Hence, we may argue that, the proposed algorithms will be more competitive for the latency-relaxed communication scenarios in practice, such as massive machine type communications (mMTC).

\section{6 | PERFORMANCE RESULTS}

In this section, we focus on demonstrating the achievable error rate performance of the multicell downlink MC DSCDMA systems employing the various resource allocation algorithms. For comparison, we consider two famous existing algorithms, namely scheme 1 and scheme 2 . In the context of the scheme 1 and scheme 2 , they respectively employ 
the greedy algorithm and the WSA algorithm based distributed subcarrier-allocation, and then they both employ the random code-allocation (RCA). In all our simulations for error rate performance, quadrature phase-shift keying (QPSK) is employed for baseband modulation. We assume all subcarriers are assumed to experience independent flat Rayleigh fading. In addition to the error rate simulations, we also evaluate the spectral-efficiency performance of the resource allocation algorithms. The number of users per cell is $K=M \times N$, where $M$ and $N$ are the number of subcarriers and the length of orthogonal DS spreading codes, respectively. For characterizing the slow fading, we assume $\mu=4$ [9, 36] for the pathloss exponent and $Y=8 \mathrm{~dB}[9,13,37]$ for shadowing effect. Further, we have the number of bins $N_{b}=4$ for the IS approach when using the EHSC.

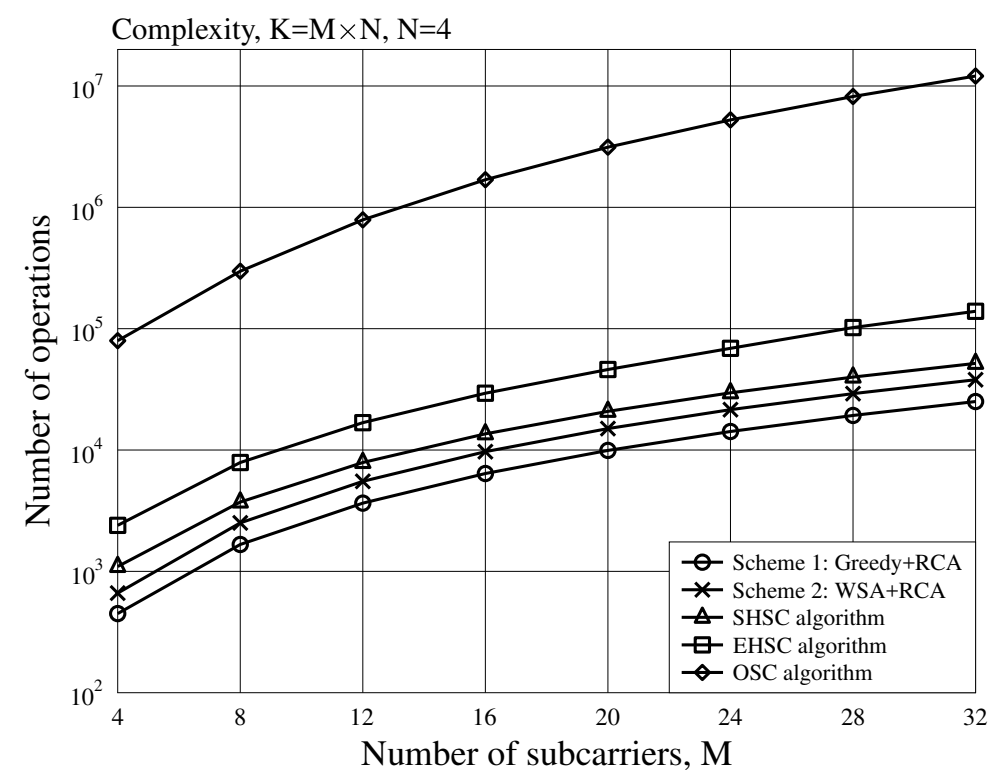

FIGURE 1 Number of comparisons required by various algorithms when $N=4$.

In Fig. 1, it compares the complexity of the algorithms with respective to different number of subcarriers employed by the multicell system. We can observe the number of comparisons required by the proposed SHSC and EHSC algorithms are always at the similar level as that of the existing low complexity algorithms which are scheme 1 and scheme 2. This observation proves that our proposed algorithms are low complexity. Fig. 2 demonstrates the BER performance of the multicell downlink MC DS-CDMA systems employing the proposed SHSC and EHSC algorithms, and the other various resource allocation algorithms. From Fig. 2, we have the following observations. First, both the proposed SHSC and EHSC algorithms can significantly outperform the existing schemes 1 and 2 in terms of the error rate performance. Second, the figure also shows that the proposed SHSC and EHSC algorithms can achieve the BER performance close to the optimal resource allocation algorithm namely OSC. Note that, for the small SNR region, the SHSC and EHSC are only slightly outperformed by the OSC algorithm, which however requires significant higher complexity than our proposed algorithms. The above two observations imply that, in the downlink multicell systems, the proposed WCF and IWE-WCF subcarrier-allocation algorithms can still significantly outperform other existing algorithms, which include the greedy algorithm for scheme 1 and the WSA algorithm for scheme 2 . In addition, the 


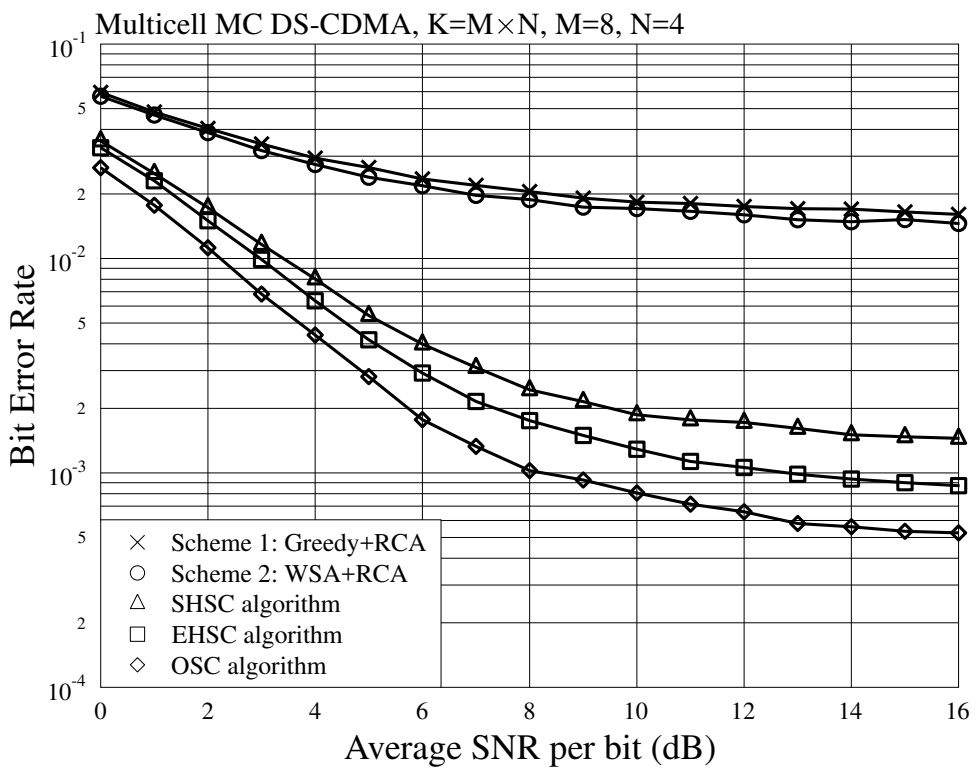

FIGURE 2 Average error rate performance of the multicell downlink MC DS-CDMA systems using the proposed SHSC and EHSC algorithms as well as the other existing resource allocation algorithms.

observations also show that, the novel code-allocation algorithms proposed by the SHSC and EHSC have very high efficiency of combating InterCl, since they can achieve the performance close to the optimal case, while significantly outperforming the RCA.

Third, from Fig. 2 we can find that the EHSC algorithm is able to obtain a better BER performance than the SHSC algorithm, since the EHSC can exploit a higher selecting diversity in terms of subcarrier- and code-allocation. However, the performance advantage of the EHSC is obtained at a cost of the increased complexity which is analyzed in Section 5.3. Fourth, from Fig. 2, we can see the BER performance achieved by schemes 1 and 2 can not be distinguished by using different subcarrier-allocation algorithms. This is because the average error rate performance of the systems is heavily affected by strong InterCI. By contrast, the proposed SHSC and EHSC algorithms have the capability of efficiently mitigating strong InterCl via code-allocation. Last, in the multicell systems, the error floors will happen when the average SNR per symbol is high, as shown in Fig. 2. This is because, in order to save backhaul resources, the proposed resource allocation algorithms only employ the InterCl factors information generated by the slow fading to operate the code-allocation for reducing the InterCl. Hence, the SHSC and EHSC algorithms are unable to fully remove the InterCI due to the lack of full InterCl information.

In Fig. 3, we focus on comparing the performance of the proposed EHSC and SHSC algorithms employed by the multicell systems. First, regardless of using the EHSC or the SHSC algorithm, the BER performance of the system can be enhanced when the number of subcarriers $M$ gets bigger, or as the number of codes $N$ gets bigger. The reason behind is, as $M$ and/or $N$ increase, the diversity order increases, and the subcarrier- and code-allocation can achieve better performance. Second, we observe that, the error performance of the EHSC can gets closer to that of the optimal one as $N$ or $M$ decreases. Third, for all the cases considered, the EHSC can achieve better performance than the SHSC, and the achievable performance gain increases, when $M$ and/or $N$ gets larger. This is mainly because the EHSC algorithm 


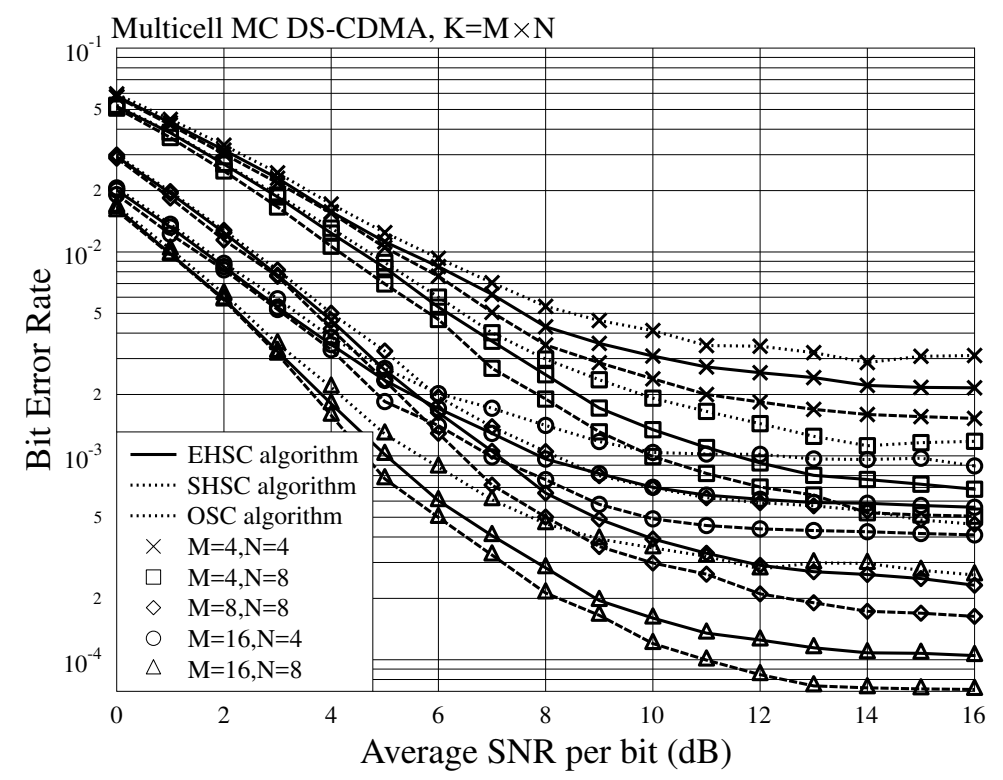

FIGURE 3 Average error rate performance of the multicell downlink MC DS-CDMA systems using the OSC, EHSC and SHSC algorithms.

exploits higher diversity than the SHSC algorithm in terms of code-allocation. The EHSC algorithm makes use of three MInterCl matrices simultaneously for code-allocation, and it can avoid more number of strong InterCl during each iteration than the SHSC which operates the allocation based on only one matrix at a time. Moreover, when comparing the scenario of $M=16$ and $N=4$ with that of $M=8$ and $N=8$, the latter one can achieve lower BER for the range of $\mathrm{SNR} \leq 6$ while higher BER for the range of $\mathrm{SNR}>6$. This observation reveals that, the system reliability is dominated by the selection diversity in subcarrier-allocation dimension, when the noise power is high. By contrast, as the noise power decreases, the InterCl will have a dominating impact on the reliability performance, especially when the number of strong InterCl is relatively high. Hence, in practical systems, we need to configure the MC DS-CDMA systems by selecting suitable numbers of subcarriers and/or codes when the communication condition, such as average SNR per symbol, varies, in order to maximize the reliability performance.

In the multicell downlink MC DS-CDMA systems, each user only suffers from the InterCl generated by its two co-subcarrier-code users. To avoid strong InterCl, a user can employ the maximum likelihood detector (MLD), which will not require too much complexity on the receiver as long as the user has two strong InterCl at most. In Fig. 4, we investigate the BER performance of the multicell systems when using different resource allocation algorithms, which are assisted by the MLD at each user. In particular, the MLD can efficiently reduce the InterCl to minimum, thereby the error floors can be removed. In Fig. 4, we can observe that, regardless of employing MLD, the proposed SHSC and EHSC can significantly outperform existing schemes 1 and 2. By comparing Fig. 4 with Figs. 2, all the corresponding results of Fig. 4 are enhanced, but the BER performance gap of the EHSC algorithm over the SHSC algorithm decreases, owing to the use of the MLD.

As known, the performance of the EHSC's code-allocation algorithm is also affected by the proposed IS approach, which determines the user priority for code-allocation. Fig. 5 evaluates how the performance of the SHSC algorithm is 


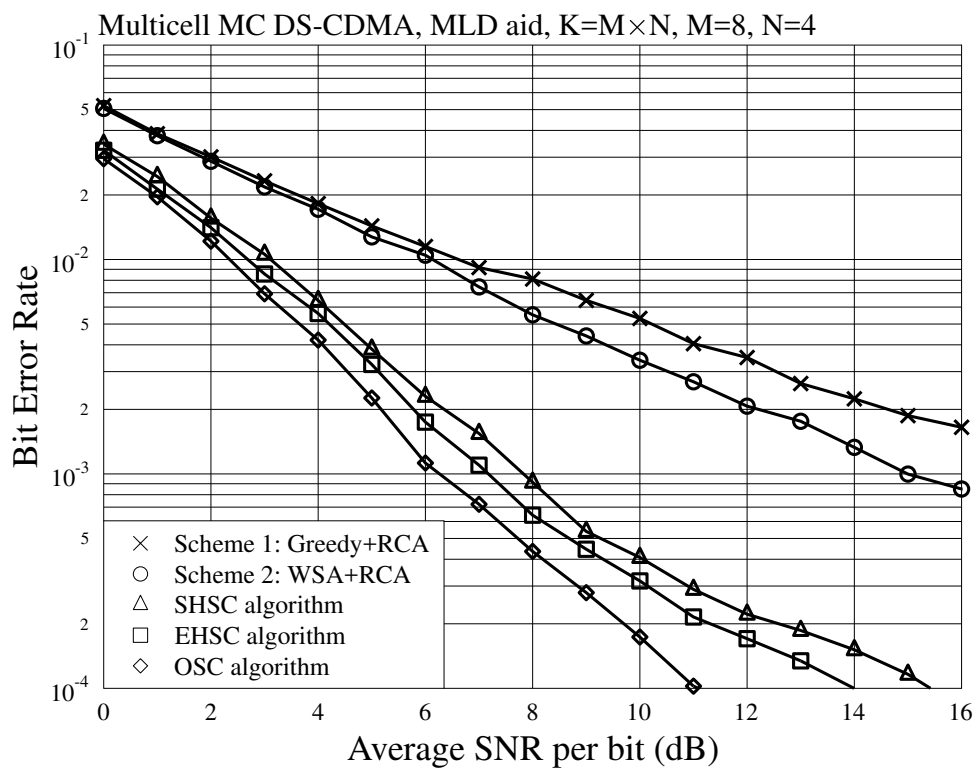

FIGURE 4 Average error rate performance of the multicell downlink MC DS-CDMA systems using different resource allocation algorithms, and assuming MLD is employed by each user.

impacted by the value of $N_{b}$ for the IS approach. Observed from Fig. 5, the average error rate decreases as $N_{b}$ increases and, eventually the performance can converge. Furthermore, as we can see, the convergence time can be reduced as the number of codes $N$ decreases. In order to implement the IS approach in practice, it is suggested to select the value of $N_{b} \leq 6$, which will not impose a big complexity. Suggested by the observations from Fig. 5 and Fig. 3, we also need to use a reasonable number of codes, which should strike a good balance among the required reliability performance, selection diversity exploited by code-allocation and the implementation complexity.

Let us now evaluate the per user reliability for the multicell system in Fig. 6. Note that, to reflect the per user reliability, it computes the cumulative distribution function (CDF) of a randomly selected user in the system, and the results are obtained from $10^{5}$ number of realizations. Again, this figure shows that the proposed SHSC and EHSC algorithms can significantly outperform the existing algorithms including scheme 1 and scheme 2 in terms of the performance metric of the per user reliability. Further, in majority regions the CDF curves for our proposed algorithms appear to be much more flat than those for scheme 1 and scheme 2. This observation implies that, the resource allocation by the proposed algorithms can significantly reduce the probability that resulting in a single user having poor link reliability, which may be caused by deep fading (by subcarrier-allocation) or strong InterCl (by code-allocation). In addition, the above observation also shows that our proposed algorithm can result in a higher fairness (in terms of reliability) for users than the existing algorithms.

Furthermore, in Fig. 7, we study the achievable spectral-efficiency performance of the multicell systems ursing the proposed SHSC and EHSC algorithms, and the other existing schemes. It is observed that, for a given SNR, the EHSC and SHSC algorithms are capable of achieving higher spectral-efficiency than the existing schemes 1 and 2. However, according to the comparison in Fig. 7, the EHSC algorithm only has a very small spectral-efficiency gain over the SHSC algorithm. Furthermore, the proposed SHSC and EHSC algorithms are able to achieve the spectral- 


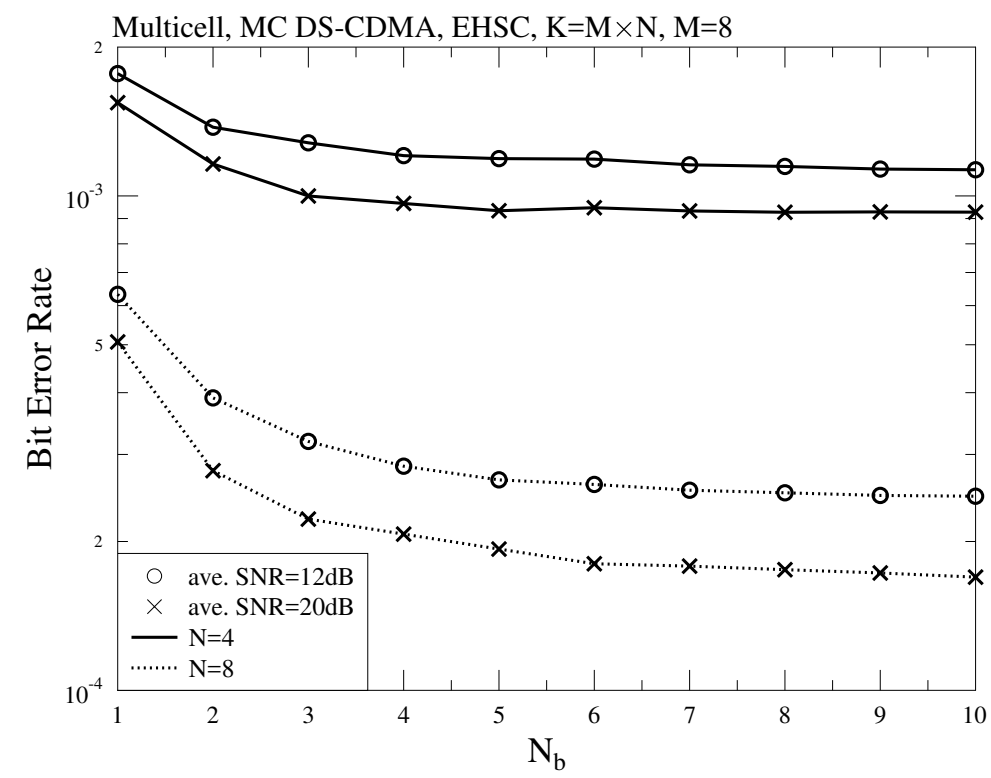

FIGURE 5 Average error rate performance of the multicell downlink MC DS-CDMA systems employing the EHSC algorithm, which is characterized by the IS approach.

efficiency performance close to that of the OSC algorithm. Fig. 7 also demonstrate that, in the multicell scenarios, the subcarrier-allocation algorithms proposed can achieve a higher performance than the other existing subcarrierallocation algorithms in terms of the spectral-efficiency. In general, considering both the error rate and the spectralefficiency performance, we can see that the SHSC and EHSC algorithms can not only achieve significant better BER performance but also yield higher spectral-efficiency than the existing schemes 1 and 2 considered.

\section{7 | CONCLUSIONS}

In this paper, we have investigated the high-reliability and low-complexity based resource allocation for future multicarrier spread spectrum systems. Motivating to minimize the implementation complexity, we have proposed a novel strategy for managing resources, where the subcarrier-allocation is independently by each BS and, then, code-allocation is implemented with the knowledge of the subcarrier-allocation. Two resource allocation algorithms, namely SHSC and EHSC, have been proposed. Under the two proposed algorithms, the BSs only require the intracell CSI for implementing subcarrier-allocation, and the BSs only exchange the InterCl information (InterCl factors) generated by the slow fading for carrying out code-allocation. Hence, both the two algorithms can be operated with relatively low complexity backhaul signaling. While the EHSC algorithm requires higher complexity than the SHSC, it can exploit a higher selecting diversity. Our performance results show that both the SHSC and EHSC algorithms significantly outperform the existing resource allocation algorithms considered, and both can achieve the performance close to the optimal resource allocation algorithm namely OSC. Furthermore, our simulation results demonstrate that the proposed algorithms do not make the trade-off between error rate performance and spectral-efficiency performance. 


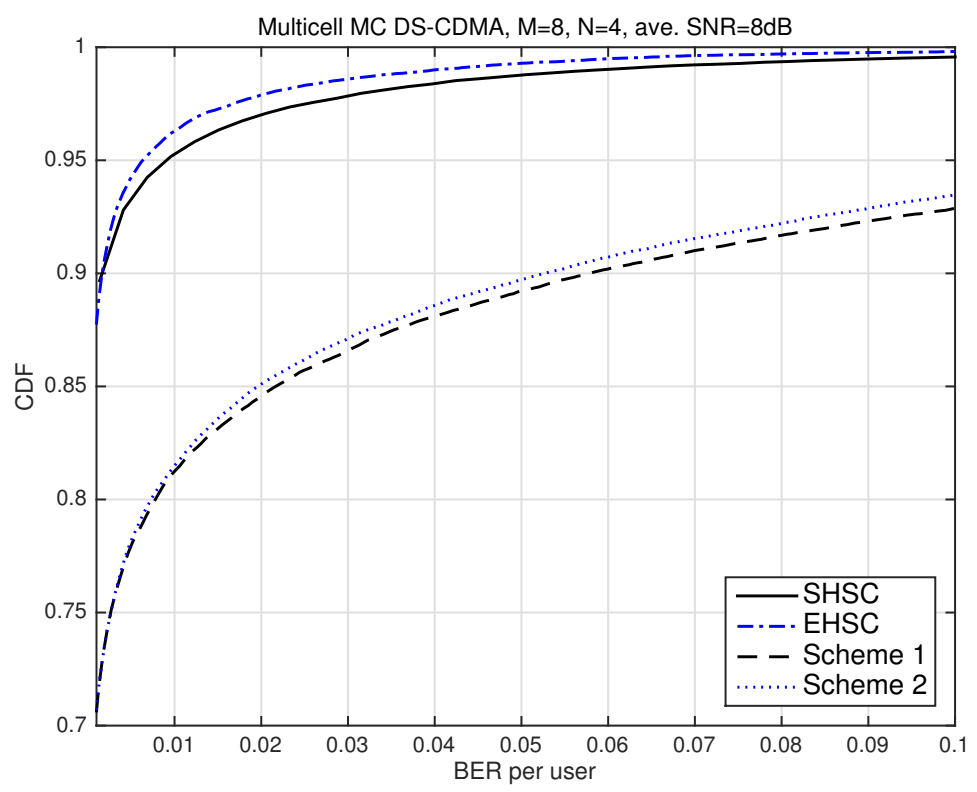

FIGURE 6 Comparison for CDF of BER per user in the multicell downlink MC DS-CDMA systems employing the proposed SHSC and EHSC algorithms as well as other resource allocation algorithms.

\section{ENDNOTES}

\section{REFERENCES}

[1] A. Masmoudi, F. Bellili, S. Affes and A. Ghrayeb, "Maximum Likelihood Time Delay Estimation From Single- and MultiCarrier DSSS Multipath MIMO Transmissions for Future 5G Networks," IEEE Transactions on Wireless Communications, vol. 16, no. 8, pp. 4851-4865, 2017.

[2] J. Zhang, L. L. Yang and L. Hanzo and H. Gharavi, "Advances in Cooperative Single-Carrier FDMA Communications: Beyond LTE-Advanced," IEEE Communications Surveys Tutorials, vol. 17, no. 2, pp. 730-756, 2015.

[3] Z. Ding, X. Lei, G. K. Karagiannidis, R. Schober, J. Yuan and V. K. Bhargava, "Maximum Likelihood Time Delay Estimation From Single- and Multi-Carrier DSSS Multipath MIMO Transmissions for Future 5G Networks," IEEE Journal on Selected Areas in Communications, vol. 35, no. 10, pp. 2181-2195, 2017.

[4] Lie Liang Yang, "Multicarrier Communications," John Wiley, 2009.

[5] J. Zhang, L. L. Yang and L. Hanzo, "Energy-Efficient Dynamic Resource Allocation for Opportunistic-Relaying-Assisted SC-FDMA Using Turbo-Equalizer-Aided Soft Decode-and-Forward," IEEE Transactions on Vehicular Technology, vol. 62, no. 1, pp. 235-246, Jan. 2013.

[6] J. Shi and L. L. Yang, "Bidirectional Worst Subchannel Avoiding Versus Best Subchannel Seeking Subcarrier-Allocation in Downlink OFDMA Systems," IEEE Transactions on Vehicular Technology, vol. 65, no. 9, pp. 7160-7172, Sept. 2016.

[7] Tao Wang and Luc Vandendorpe, "Iterative Resource Allocation for Maximizing Weighted Sum Min-Rate in Downlink Cellular OFDMA Systems," IEEE Transactions on Signal Processing, vol. 59, no. 1, pp. 223-234, Jan. 2011. 


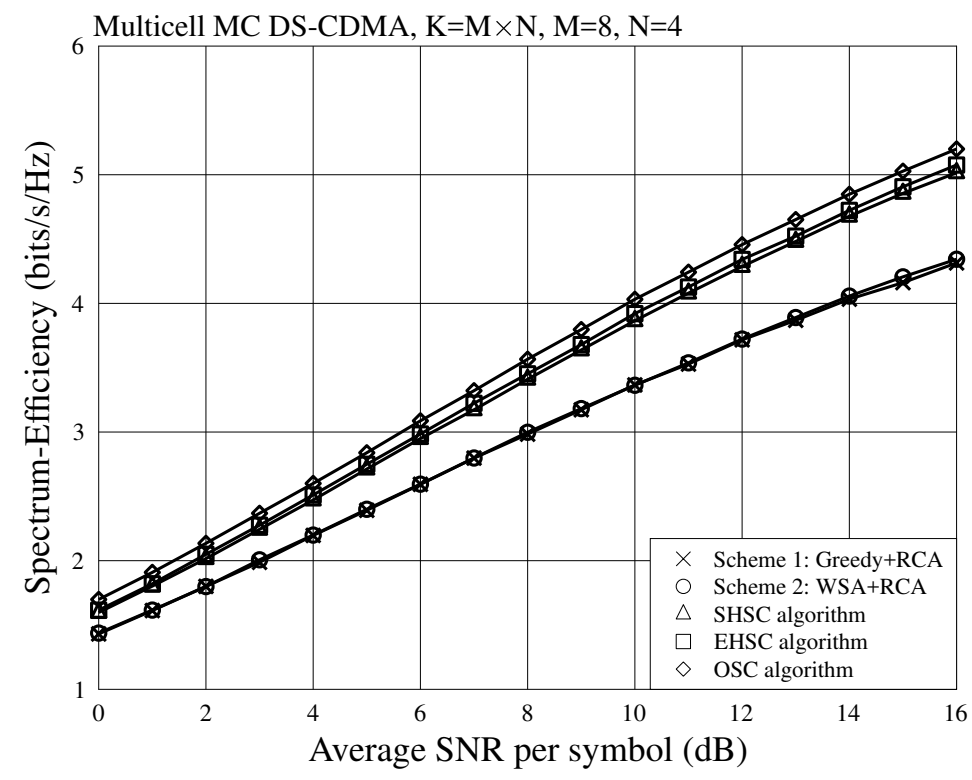

FIGURE 7 Spectral-efficiency performance of the multicell downlink MC DS-CDMA systems employing the proposed SHSC and EHSC algorithms as well as other resource allocation algorithms.

[8] D. W. K. Ng, E. S. Lo and R. Schober, "Energy-Efficient Resource Allocation in Multi-Cell OFDMA Systems with Limited Backhaul Capacity," IEEE Transactions on Wireless Communications, vol. 11, no. 10, pp. 3618 - 3631, Oct. 2012.

[9] L. Venturino, A. Zappone, C. Risi and S. Buzzi, "Energy-efficient Scheduling and Power Allocation in Downlink OFDMA Networks with Base Station Coordination," IEEE Transactions on Signal Processing, vol. 14, no. 1, pp. 1-14, Jan. 2015.

[10] E. Baktash, M. Rasti and E. Hossain, "Resource Allocation for Dynamic Intra-Cell Subcarrier Reuse in Cooperative OFDMA Wireless Networks, IEEE Transactions on Mobile Computing, vol. 14, no. 7, pp. 1475-1489, July 2015.

[11] B. Ozbek, D. Le Ruyet and M. Pischella, "Adaptive Reduced Feedback Links for Distributed Power Allocation in Multicell MISO-OFDMA Networks," IEEE Wireless Communications Letters, vol. 3, no. 2, pp. 141-144, April 2014.

[12] M. Moretti, A. Todini, A. Baiocchi and G. Dainelli, "A Layered Architecture for Fair Resource Allocation in Multicellular Multicarrier Systems," IEEE Transactions on Vehicular Technology, vol. 60, no. 4, pp. 1788-1798, May 2011.

[13] Y. Yu, E. Dutkiewicz, X. Huang and M. Mueck, “Downlink Resource Allocation for Next Generation Wireless Networks with Inter-Cell Interference," IEEE Transactions on Wireless Communications vol. 12, no. 4, pp. 1783-1793, April 2013.

[14] Jia Shi, "Cooperative and Resource Allocation in Relay and Multicarrier Systems", University of Southampton, School of Computer Science and Electronics, PhD Thesis.

[15] Q. Qu, L. B. Milstein and D. R. Vaman, "Cognitive Radio Based Multi-User Resource Allocation in Mobile Ad Hoc Networks Using Multi-Carrier CDMA Modulation," IEEE Journal on Selected Areas in Communications, vol. 26, no. 1, pp. 70-82, Jan. 2008.

[16] C.-W. Chang and L.-C. Wang, "Effects of Subcarrier Power Allocation on an Interference Avoidance Code Assignment Strategy for Multirate MC-DS-CDMA Systems," IEEE Transactions on Vehicular Technology vol. 57, no. 3, pp. 1513-1526, May 2008. 
[17] C.-W. Chang and L.-C. Wang, "A Low Interference Time-Slicing Code Assignment for the 2D-Spread MC-DS-CDMA Systems," IEEE Vehicular Technology Conference (VTC2009-Spring), pp. 1-5, April 2009.

[18] C.-W. Chang and L.-C. Wang, "A Novel Interference-Avoidance Code Reassignment for Downlink Two-DimensionalSpread MC-DS-CDMA Systems With Power Control," IEEE Transactions on Vehicular Technology vol. 59, no. 6, pp. 31043108, July 2010.

[19] L. Loyola and T. Miki, "Speed-aware multicarrier transmission and resource allocation scheme for the uplink channel of highly mobile networks," IEEE Topical Conference on Wireless Communication Technology, pp. 117-118, Oct. 2003.

[20] Z. Wang and Q. Peng and L. B. Milstein, "Multi-User Resource Allocation for Downlink Multi-Cluster Multicarrier DS CDMA System," IEEE Transactions on Wireless Communications vol. 10, no. 8, pp. 2534-2542, Aug. 2011.

[21] Z. Wang, D. Yang and L. B. Milstein, "Multi-User Resource Allocation for a Distributed Multi-Carrier DS-CDMANetwork," IEEE Transactions on Communications, vol. 60, no. 1, pp. 143 - 152, 2012.

[22] G. Femenias, F. Riera-Palou, X. Mestre and J. J. Olmos, "Downlink Scheduling and Resource Allocation for 5G MIMOMulticarrier: OFDM vs FBMC/OQAM," IEEE Access vol. 5, pp. 13770-13786, 2017.

[23] Chieh Yuan Ho and Ching-Yao Huang, "Non-Cooperative Multi-Cell Resource Allocation and Modulation Adaptation for Maximizing Energy Efficiency in Uplink OFDMA Cellular Networks," IEEE Wireless Communications Letters, vol. 1, no. 5, pp. 420-423, 2012.

[24] Sung-Yeon Kim, Jeong-Ahn Kwon and Jang-Won Lee, "Sum-Rate Maximization for Multicell OFDMA Systems," IEEE Transactions on Vehicular Technology, vol. 64, no. 9, pp. 4158-4169, 2015.

[25] K. Kim and J. Kim, "A 2-D subcarrier allocation scheme for capacity enhancement in a clustered OFDM system," IEICE Transactions on Communications, vol. E90-B, pp. 1880 - 1883, July 2007.

[26] K. A. D. Teo, Y. Otani and S. Ohno, "Adaptive subcarrier allocation for multi-user OFDM system," IEICE Transactions on Communications, vol. E89-A, pp. 3131 - 3137, July 2006.

[27] S. H. Ali and V. C. M. Leung, "Dynamic frequency allocation in fractional frequency reused OFDMA networks," IEEE Transactions on Wireless Communications vol. 8, no. 8, pp. 4286 - 4295, Aug. 2009.

[28] H. Galeana-Zapien and R. Ferrus, "Design and Evaluation of a Backhaul-Aware Base Station Assignment Algorithm for OFDMA-Based Cellular Networks," IEEE Transactions on Wireless Communications vol. 9, no. 10, pp. 3226-3237, Oct. 2010.

[29] A. J. Viterbi, A. M. Viterbi and E. Zehavi, "Other-cell Interference in Cellular Power-controlled CDMA," IEEE Transactions on Communications, vol. 42, no. 234, pp. 1501 - 1504, Feb. 1994.

[30] Dimitri P. Bertsekas, “onlinear Programming," Athena Scientific, Nashua, USA, 2003.

[31] Y. Liang, V. V. Veeravalli and H. V. Poor, "Other-cell Interference in Cellular Power-controlled CDMA," IEEE Transactions on Information Theory, vol. 53, no. 10, pp. 3432 - 3453, Oct. 2007.

[32] T. Liu, C. Yang and L.-L. Yang, "A low-complexity subcarrier-power allocation scheme for frequency-division multipleaccess systems," IEEE Transactions on Wireless Communications vol. 9, no. 5, pp. 1564 - 1570, May 2010.

[33] J. Shi and L.-L. Yang, "Novel subcarrier-allocation schemes for downlink MC DS-CDMA systems," IEEE Transactions on Wireless Communications, vol. 13, no. 10, pp. 5716-5728, Oct. 2014.

[34] P. Li and S. Guo and W. Zhuang and B. Ye, "On Efficient Resource Allocation for Cognitive and Cooperative Communications," IEEE Journal on Selected Areas in Communications vol. 32, no. 2, pp. 264-273, 2014.

[35] H. W. Kuhn, "The Hungarian method for the assignment problem," Naval Research Logistics Quarterly, vol. 2, pp. 83 - 97 , 1995. 
[36] Andrea Goldsmith, "Wireless Communications," Cambridge University Press, 2005, U.K.

[37] Kae Won Choi, E. Hossain and Dong In Kim, "Downlink Subchannel and Power Allocation in Multi-Cell OFDMA Cognitive Radio Networks," IEEE Transactions on Wireless Communications, vol. 10, no. 7, pp. 2259-2271, July, 2011. 